\title{
Uber die Größe der pleistozänen Pferde der Caballus-Gruppe in Europa und Nordasien
}

\author{
Von O. Sickenberg, Hannover
}

Mit 5 Abbildungen im Text

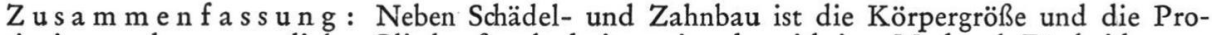
portionierung des metapodialen Gliedmaßenabschnittes ein sehr wichtiges Merkmal. Für beide werden Beziehungen zu bestimmten Temperaturverhältnissen des jeweiligen Lebensraumes und zu dessen landschaftlichen Charakter wahrscheinlich gemacht. Ihre Ermittlung vermag auch wertvolle Hinweise in taxionomischen und stammesgeschichtlichen Fragen zu geben, als Merkmale allein genommen können sie aber nicht als Grundlage einer taxionomischen Ordnung dienen, da Angehörige verschiedener Formenkreise annähernd gleiche Körpergröße und -proportionierung zeigen. Im Gegensatz zu den Eseln, Halbeseln, Zebras, aber auch zum Przewalski-Pferd und wahrscheinlich auch zu den Pferden des Equus sanmeniensis - Formenkreises sind sämtliche pleistozänen Pferde der Untergattung Caballus in der eurosibirischen Region unabhängig von der Körpergröße „schwer" gebaut, d.h. sie besitzen kurze, verhältnismäßig plumpe Metapodien. Sie waren Wald-, Bergland oder Tundrabewohner und nicht in trockenwarmen oder -kalten, ebenen Steppengebieten beheimatet. Das gleiche dürfte auch für die altquartären Pferde des „Allohippus“Formenkreises anzunehmen sein. Eine Gruppierung nach drei Größenkategorien kann vorgenommen werden (Groß-, Mittel- und Kleinpferde). Groß- und Kleinpferde gehören möglicherweise jeweils zu einer taxionomischen Einheit, die Mittelpferde sind dagegen ein Formengemenge. $\mathrm{Zu}-$ mindest von der Holstein-Warmzeit an erscheinen in Mittel- und Osteuropa, sowie in Nordasien große Pferde in den Tiergesellschaften der wärmeren Phasen, das Auftreten von Klein- und Kleinstpferden steht im Zusammenhang mit dem Vorherrschen ungünstiger Klimabedingungen.
\end{abstract}

$\mathrm{Sum}$ m r y. Apart from skull- and tooth-structure, the size of body and the proportioning of the metapodial section of limbs are very important characteristics. Both criteria are said to be related with certain conditions of temperature of the respective living area as well as with its specific scenery. Moreover, their investigation can yield valuable indications as to taxionomy and phylogeny. Merely taken as characteristics, they do not suffice to serve as a base for a taxionomic classification, since members of different type-series show approximately the same size and proportioning of body. In opposition to donkeys, zebras and even to the Przewalski horse as well as probably to the horses of the Equus sanmeniensis series, all pleistocene horses of the Caballus subgenus in the eurosiberian region are - independant of the size of body - "heavily" built, that means they have got short, relatively clumsy metapodial bones. They were dwellers in woods, in highlands or in the tundra and not in dry-warm or dry-cold plain steppes. The same may be assumed for the old-quaternary horses of the "Allohippus" series. A classification into three categories can be carried through (large-, medium- and small-sized horses). Large- and small-sized horses may possibly belong to one taxionomic unity each. The mediumsized horses on the other hand represent a product of an intermingling of types. Beginning with the Holstein-Warm-Period at the latest, in Central and Eastern Europe as well as in Northern Asia, large horses occur in the association of animals of the warmer phases. The occurrence of small and smallest horses is connected with the prevailing of unfavourable climatic conditions.

\section{I}

Wohl alle Bearbeiter sind sich über den nach wie vor unbefriedigenden Stand unserer Kenntnisse über die pleistozänen Equiden einig. Da wir uns nicht einmal über Taxionomie und Nomenklatorik hinreichend klar sind, kann nicht erwartet werden, wir vermöchten bestimmte Aussagen über die phyletischen Beziehungen, über die stratigraphische Reichweite einzelner Formen, über Lebensraum und Lebensgewohnheiten, über die geographische Verteilung und über vielleicht eingetretene Arealverschiebungen zu treffen. Der Ursachen, die dafür verantwortlich zu machen sind, sind mehrere. Funde ganzer Skelette oder größerer zusammenhängender Skeletteile, ja selbst nur gut erhaltener Schädel sind selten. Einigermaßen bekannt sind nur die Equidenreste bestimmter Fundorte in Deutschland, Schweiz und Österreich sowie in Frankreich, England und den Niederlanden. In den letztgenannten drei Ländern erfolgten in letzter Zeit allerdings hauptsächlich nur 
Veröffentlichungen über altquartäre Pferde. In Schweden sind es wieder nur die holozänen Wildpferde, die, allerdings sehr gründlich, untersucht wurden. Der gesamte Süden und Südosten Europas stellt hinsichtlich der Pferde praktisch eine terra incognita dar. Dank der umfassenden Zusammenstellung Gromova's und älterer russischer Arbeiten liegen einige Angaben über die Pferde des gewaltigen eurasiatischen Raumes nördlich der Gebirgsländer vor. Dabei fehlen monographische Bearbeitungen aus neuerer Zeit, sei es aus regionaler oder stratigraphischer Sicht, allenthalben. Die Beurteilung fossilen Materials wird weiter durch den Umstand sehr erschwert, daß wir über Ausmaß und Art der Variabilität der Hartteile der lebenden Wildpferde einschl. des Przewalski-Pferdes, gewonnen an einem genügend großen Material, keineswegs zufriedenstellend unterrichtet sind. Ungenügende Materialkenntnis allein bedingt aber nicht die Schwierigkeiten. Die gesamten Pferde neigen vom Beginn ihrer Stammesgeschichte kaum dazu, ihren Bauplan wesentlich abzuändern. Eine gewisse Starrheit ist unverkennbar, die sich natürlich besonders im Laufe ihrer späteren, also jungtertiären und quartären Geschichte bemerkbar macht. Das Formbildungsvermögen reicht im Gegensatz zu anderen, plastischeren Stämmen nicht hin, aus dem Grundstock in morphologischer und biologischer Hinsicht eigenständige Zweige hervorzutreiben, es erschöptt sich sozusagen in einer vielfältigen Abwandlung des "Typus“. Es ist groß genug, innerhalb der vergleichsweise wenigen, gut abgrenzbaren Formenkreise eine offenbar große Anzahl taxionomischer Kategorien niederer und niedrigster Rangordnung zu schaffen, zu schwach aber, um es noch einmal zu sagen, entscheidend über den Rahmen des gegebenen hinauszuwachsen oder ihn gar zu sprengen. Die unverkennbare „Neigung“ der späten Equiden, auf offensichtliche Änderungen der klimatischen und damit landschaftlichen Bedingungen ihrer Lebensräume höchstens nur mit Bildung von Klein- und Kleinstarten zu antworten, steht damit in Zusammenhang. Diese richtig zu erkennen und abzugrenzen, ihre Entstehung aus endogenen oder exogenen Ursachen heraus verstehen zu wollen, stellt daher den Palaeozoologen vor äußerst schwierige Probleme. Was er an seinem und am Material aus der Gegenwart feststellen kann, ist dies: Durchgreifende, klar faßbare strukturelle Unterschiede im Schädelbau, in der Bezahnung und bei den übrigen Skelettelementen fehlen selbst zwischen den Hauptformenkreisen, die äußere Konfiguration des Schädels und die Proportionierung seiner Teile wird aber erheblich variiert, das Gebiß zeigt zwar von Population zu Population ein jeweils eigenes Gepräge, nur sehr wenige Merkmale scheinen aber einigermaßen gattungs- oder artkonstant zu sein. Am auffälligsten sind die Unterschiede in der Gesamtgröße der Tiere und die Proportionierung der einzelnen Teile des Bewegungsapparates.

Das Ziel der nachstehenden Studie ist, die Größe der Pferde des Fleistozäns Europas und des nördlichen Asiens in Beziehung auf Raum, Zeit und Umweltsbedingungen zu untersuchen.

$\mathrm{Da}$ fossile Belege dieses Alters aus der Zebra-Halbesel- und Esel-Gruppe entweder sehr spärlich sind oder überhaupt fehlen, gebot sich von selbst die Beschränkung auf die Caballus-Gruppe, d. h. auf die Gattung Equus s. str., wobei vor allem deren jungpleistozäne Vertreter berücksichtigt wurden. Die altpleistozänen Formen bleiben etwas außerhalb der Betrachtung, einmal weil ihre Zugehörigkeit zur Caballus-Gruppe bei den Angehörigen des „Stenonis-Kreises“ im weiteren Sinne zweifelhaft ist, zum anderen, weil auswertbare Reste noch seltener als im jüngeren Quartär sind. $\mathrm{Da}$ von den Extremitätenknochen unter fossilem Material die Metapodien am häufigsten sind und den besten Erhaltungszustand zeigen, wurden diese allein der Untersuchung zu Grunde gelegt. Vorausgesetzt wird dabei allerdings, daß die Länge von Mittelhand und -fuß Rückschlüsse auf die Gesamtgröße erlaubt, wozu allerdings bemerkt werden muß, daß bei gewissen Formen bei eintretender "Verplumpung" (Längenabnahme bei gleichbleibender Breite) vorzugsweise nur der metapodiale Extremitätenabschnitt betroffen wird (HoFStEtTen 1952). Die plumpen Kleinpferde waren daher in Wirklichkeit etwas größer als 
man nach den Längen ihrer Metapodien vermeinen könnte. Zur Feststellung der jeweiligen Größenverhältnisse dienten die größte Länge und die Breite in der Diaphysenmitte der einzelnen Exemplare. Die Angaben wurden fast ausnahmslos der Literatur entnommen, besonders aus den Arbeiten von Schwarz 1927 und Gromova 1949, die allerdings selbst wieder zu einem guten Teil auf die Messungen früherer Bearbeiter zurückgehen. Ungenauigkeiten der verschiedensten Art schleichen sich damit natürlich ein und beeinträchtigen die Ergebnisse (verschiedene Meßmethoden, Hereinnahme der Meßzahlen jugendlicher, als solche aber nicht gekennzeichneter Individuen in die Maßtabellen, mangelnde Berücksichtigung des Erhaltungszustandes usw.). Dies mußte in Kauf genommen werden, um überhaupt verfahren zu können, wenn solche Fehler auch angesichts der spärlichen Stückzahl schwerer wiegen als bei reicher Materialfülle. Das Gesamtbild mag dadurch an der einen oder anderen Stelle vielleicht etwas verzerrt, bis zur Unkenntlichkeit verfälscht aber nicht werden. Auch die sexuellen Unterschiede, die sich zumindest beim Hauspferd bei den Metapodien feststellen lassen (KunKaitis nach Lundholm 1949) konnten naturgemäß nicht berücksichtigt werden. Die Maßzahlen wurden in Diagramme eingetragen, welche zunächst die Position jedes einzelnen Exemplares nach Gesamtlänge und Diaphysenmitten-Breite erkennen lassen. Außerdem wurde von den jeweils extremsten Werten ausgehend versucht, Rechtecksfelder zu konstruieren, um den Variationsbereich bestimmter Gruppen graphisch darzustellen. Durch die Überbetonung der Breite werden zwar die Proportionen verzerrt, zugleich bestimmte Verhältnisse aber auch verdeutlicht. Selbstredend bedeutet ein solches Verfahren nur eine sehr grobe Annäherung an die Wirklichkeiten, da die Stückanzahl, welche diesen Konstruktionen zu Grunde liegt, jeweils sehr verschieden und in keinem der Fälle ausreichend genug ist. Von keiner echten Population - die ja sowieso nur bei rezenten Formen untersuchbar ist - liegt genügend großes Material vor, das erlauben würde, Größe und Konfiguration des Variationsfeldes festzustellen und die Anordnung der einzelnen Exemplare in diesem einwandfrei zu erkennen. Aus theoretischen Erwägungen heraus kann als sicher gelten, daß sich die den einzelnen Exemplaren entsprechenden Marken nicht gleichmäßig über das Feld verteilen, sondern sich entlang einer oder zweier Achsen, - bei Bestehen merklicher sexueller Unterschiede, anordnen. Ein Überschneiden der einzelnen Felder in der graphischen Darstellung bedeutet daher noch keineswegs, daß sich die Werte realiter überschneiden, dies darf erst bei bedeutenderem Deckungsgrade zweier Felder angenommen werden.

Aus den gleichen Gründen wurde auch auf eine rechnerisch-variationsstatistische Behandlung der Materialien verzichtet. Über das, was durch Maßangaben genau belegbar ist, wurde nicht hinausgegangen, denn die in der Literatur sehr häufigen, allgemein gehaltenen Angaben wie "große Form“, „kleines Pferd“ sind zu subjektiv gefärbt, um viel von Nutzen zu sein.

Im Rahmen der vorliegenden Studie bot sich bei dem gegenwärtigen Stand der Dinge keine andere Möglichkeit, als sich der konventionellen Taxionomie und Nomenklatorik zu bedienen. $\mathrm{Da}$ die Auffassungen darüber, was als Art und was als Unterart anzusehen sei, bei den einzelnen Autoren weit auseinandergehen, die Zeit für Entscheidungen auch noch nicht reif ist, erscheinen die einzelnen, bisher benannten Formen durchgehend als Arten. Von den verschiedenen Untergattungen der Großgattung Equus sind in den Untersuchungskreis nur folgende einbezogen: Caballus im Sinne Dietrichs, Asinus, Hemionus und Onager (hier zusammengefaßt zur Hemionus-Gruppe), Hippotigris, Dolichohippus, Zebra und Quagga (hier zusammengefaßt zur "Hippotigris"-Gruppe), Amerbippus und schließlich Allohippus, die letztgenannte, derzeit noch nicht genauer zu kennzeichnende und abgrenzbare Untergattung gebraucht als Sammelbezeichnung für alle altquartären Equiden, die nicht eindeutig zur Untergattung Caballus oder zu einer der anderen aufgeführten Untergattungen gehören (Simpson 1945, Hofstetter 1952, DietRICH 1950). Die oben genannte Bemerkung bezüglich Taxionomie und Nomenklatorik 
bezieht sich vor allem auf die Untergattung Caballus. Unter ihren zahlreichen "Arten" scheint mir nur als selbständige taxionomische Einheit das Pferd aus den Mosbacher Sanden (=C.mosbachensis v. REICHENAU) gesichert, alles andere erscheint derzeit sowohl taxionomisch, wie nomenklatorisch unsicher (E.germanicus NEHRING, E. steinheimensis v. Reichenau, E.taubachensis v. Reichenau, E. chosaricus Gromova, E. missi M. Pavl., E. woldrichi Antonius usw.); von einer wirklichen Ordnung sind wir noch weit entfernt.

Man wird verstehen, daß angesichts dieser Sachlage das gewählte Thema nur mit den größten Vorbehalten angegangen werden kann. Ein derartiger Versuch darf überhaupt nur in der Zuversicht unternommen werden, daß jede kritische Materialsichtung im Hinblick auf eine bestimmte Frage von Nutzen sein wird, wenn nicht von anderem, so doch wenigstens von dem einen, diese Frage schärfer und enger zu fassen, um andere Untersuchungen anzuregen und damit die Möglichkeit zu eröffnen, eine der Wirklichkeit gemäße Antwort zu finden. Dies möge bedacht werden, wenn hier entschieden mehr Probleme gestellt als gelöst erscheinen, was mit gewissem Recht als enttäuschend empfunden werden mag. Ein umfassender Versuch, an Hand von fossilem Material (Metapodien) den Größenverhältnissen der pleistozänen Equiden Europas nachzugehen, um damit zu gewissen Schlußfolgerungen zu kommen, wurde erst einmal gemacht (ScHwarz 1927). Er war ziemlich berechtigter Kritik ausgesetzt, da chronologische Daten nicht entsprechend berücksichtigt und die Verschiedenartigkeit der oekologischen Verhältnisse vernachlässigt wurden. Die Ergebnisse, namentlich im taxionomischen Bereich, ruhen daher zwangsläufig auf mehr als schwankem Boden.

\section{II}

Die Hauptgruppen der pleistozänen und holozänen Equiden

Material: Me t a carpa $1 \mathrm{e}$

(Diagramm 1a und $1 b$ )

1. Caballus-Gruppe einschl. Allohippus:

Umfaßt nahezu das gesamte veröffentlichte, mit einiger Sicherheit auf die CaballusGruppe beziehbare pleistozäne Material Europas und Nordasiens (einschl. E. sanmeniensis TeILh. DE JARD. et PIV., $\sim 200$ Exempl.) sowie E. przewalski Pol. (8 Exempl.).

2. Hemionus-Gruppe:

H. hemionus Pall. u. H.onager BoDD., teils fossil aus $\mathrm{N}$-Asien, teils subfossil und rezent, insges. 11 Exempl.

3. Asinus-Gruppe:

Asinus asinus L., A. somaliensis, A. bydruntinus REg., insgesamt 13 Exempl. Ein von Pomel veröffentlichtes Exemplar (n. Gromova) aus dem Neolithikum Nordafrikas wurde nicht berücksichtigt; die extreme Schlankheit dieses Mtc würde das Feld bedeutend nach links erweitern. Ebenso wurde ein sehr großes Exemplar v. A. hydruntinus, (Fundlok. Tiraspol), dessen Zugehörigkeit z. A.h. m. E. fraglich ist, nicht berücksichtigt.

4. Hippotigris-Gruppe (Hippotigris s. 1.):

Hippotigris mauretanicus Poм., H. boebmi, H. burchelli, H. zebra, insges. 4 Exempl.

5. Amerhippus-Gruppe:

A. Andium Wagn.-Branco, A. neogaeus Lund, A. curvidens Owen, insges. 51 Exempl.

Metatarsale

1. Caballus-Gruppe, wie oben, 250 Exemplare.

2. Hemionus-Gruppe, wie oben, insgesamt 11 Exemplare aber z. T. von anderen Fundorten als die Metacarpalia). 

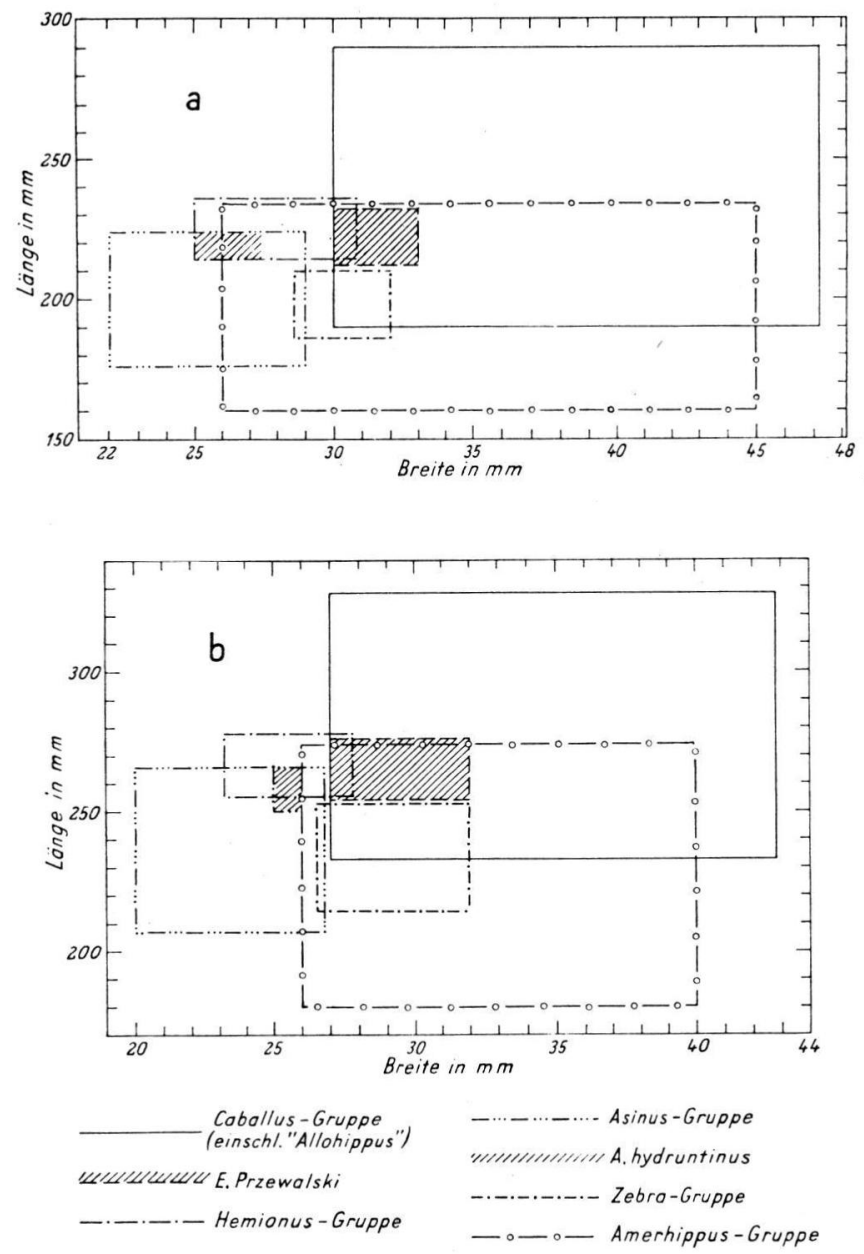

Abb. 1. Die Hauptgruppen der pleistozänen und rezenten Pferde (Europa, Asien, Afrika, Südamerika). a: Metacarpale, b: Metatarsale.

3. Asinus-Gruppe:

Wie oben, insgesamt 11; auch hier wurden nur die mitteleuropäischen Exemplare von A. bydruntinus berücksichtigt, da bei den größerdimensionierten osteuropäischen Vertretern Fehlbestimmungen vorliegen können (Verwechslung mit Angehörigen der Hemionus-Gruppe!).

4. Hippotigris-Gruppe:

H. mauretanicus Pом., H.oldowayensis Hopw., H.boehmi, H.burchelli, H.zebra, insgesamt 5 Exemplare.

5. Amerhippus-Gruppe:

A. Andium Wagn.-Branco, A. Martinei SpIllm., A. Santae-Elenae Spillm., A.neogaeus Lund, A. insulatus C. Amegh., A.curvidens Owen, insgesamt 55 Exempl.

Die Diagramme wurden aufgestellt, um die größenmäßigen Beziehungen zwischen den wichtigsten altweltlichen Untergattungen zu ermitteln. Die nordamerikanischen Quartärpferde wurden also nicht mit einbezogen, die südamerikanischen Vertreter der Unter- 
gattung Amerbippus erscheinen aber zu bestimmten Vergleichszwecken in den Diagrammen.

Folgende Feststellungen ergeben sich:

1. Die einzelnen Felder überschneiden sich zwar, die einzelnen Gruppen als solche können aber mit hinreichender Deutlichkeit als selbständige Einheiten erkannt werden.

2. Zwei Hauptgruppen zeichnen sich ab: Die eine umfaßt kleine bis mittelgroße Formen mit schlanken Metapodien; dazu zählen die Hemionus-, die Asinus- und die Hippotigris-Gruppe, am schlanksten sind die Esel, plumper die Zebras, die Halbesel vermitteln, sind aber größer als die beiden (Asinus hydruntinus reiht sich in das HemionusFeld ein. Die sichere Bestimmung einzelner isolierter Metapodien von A. bydruntinus oder H. spec. scheint mir daher auf unüberwindliche Schwierigkeiten zu stoßen). Zur anderen Hauptgruppe gehören die echten Pferde einschließlich Allohippus und die Arten der Gattung Amerhippus. Es sind sehr kleine bis sehr große Pferde mit mittelschlanken bis sehr plumpen Metapodien (über das Przewalski-Pferd s. u.).

3. Die Proportionsverhältnisse sind bei den Metatarsalien "gefestigter", bei den Metacarpalia ist offenbar im allgemeinen der Spielraum für den Schlankheitsgrad größer (Ausnahme E. przewalski?).

\section{III}

Um für den weiteren Untersuchungsgang einen Ausgangspunkt zu finden, mußte versucht werden, an Hand eines einigermaßen homogenen Materials über Umfang und Art der Größenvariabilität zumindest einiger weniger der in Frage kommenden Formen bestimmtere Vorstellungen zu gewinnen. Wenn eingangs erklärt wurde, es ließen sich so gut wie keine gesicherten taxionomischen Einheiten aufstellen, so soll dies nicht bedeuten, es hätte solche überhaupt nicht gegeben. Im Gegenteil, es besteht aller Grund zur Annahme, daß die Untergattung Caballus unter allen die formenreichste war und in eine große Anzahl zumindest von "guten“ Unterarten und Rassen zerfiel. Ihre Erkennung und Abgrenzung bereitet aber derzeit fast unüberwindliche Schwierigkeiten, aus Gründen, die gleichfalls schon eingangs dargelegt wurden. Entweder handelt es sich beim fossilen Material um Eirizelfunde oder das Material ist zwar umfangreicher, aber wahrscheinlich häufig uneinheitlich, denn bei fast allen älteren Aufsammlungen wurden die Knochen eines Fundortes nicht genügend nach den einzelnen Fundschichten getrennt und dadurch nicht selten verschiedenartiges vermengt. Die Fundlager, Höhlenablagerungen, Terrassenschotter und Lößmassen sind meist, wie sich immer mehr herausstellt, recht komplexer Natur und nur selten zeitliche und ökologische Einheiten. Auch wenn eine wirkliche Einheit gegeben erscheint, so muß in Rechnung gesetzt werden, daß möglicherweise zu gleicher Zeit zwei oder mehr Formen den gleichen Raum besetzten, wie man dies von den heutigen Zebras kennt. Um aber mit Sicherheit ein derartiges Kollektiv in seine Bestandteile auflösen zu können, dazu bedarf es eines größeren Materials, als in der Regel von eurasiatischen Fundplätzen zur Verfügung steht. Im Grunde genommen entspricht keine der bisherigen Aufsammlungen, von einer Ausnahme abgesehen, den anzulegenden Maßstäben, eine wenig ermutigende Feststellung, die aber um der Klarheit willen notwendig erscheint. Um nichts besser steht es bei dem rezenten Vertreter der Untergattung, bei E. przerwalski PoL., das bekanntlich nur noch in wenigen Exemplaren als Wildform vorkommt und daher in keiner Sammlung der Welt in reichen Serien vertreten ist. Unsere Kenntnis beschränkt sich daher auf das, was die Untersuchung nur weniger Exemplare ergibt. Außerdem kann eine wenn auch geringe Beimengung von Hauspferdblut bei den heute lebenden Wildpferdbeständen nicht ganz ausgeschlossen werden (Mонr 1959). Ein anderes subrezentes Wildpferd, der Tarpan (E. gmelini ANT.) kann zwar auf Grund verschiedener Erwägungen als ehemals existent angenommen werden, als taxionomische Kategorie ist es aber das Ergebnis einer Konstruktion, hinter der eine wahrscheinliche, aber keineswegs 
gesicherte und zweifelsfreie Realität steht ${ }^{1}$ ), auf deren in Sammlungen befindlichen Belegen bei Untersuchungen zurückgegriffen werden kann. In den Tabellen bei Gromova (1949) ist daher der Tarpan nur mit einem Exemplar vertreten.

Bei der geschilderten Lage blieb mithin kein anderer Weg offen, als das Material verschiedener Fundpunkte, soweit es der leider immer sehr bescheidene Umfang ïberhaupt noch zuläßt, zum Aufbau des Diagrammes (2a u. b) zu verwerten. Über die chronologischen und klimatischen Daten der einzelnen Fundlokalitäten bzw. -komplexe gibt der Anhang S. 120) Aufschluß, auf den auch für die später folgenden Ausführungen zu verweisen ist.

Equus przerealski PoL., Rezent;

Stückzahl: Metacarpalia: 8, Metatarsalia: 8 (nach Gromova 1949).

E. gmelini ANT., Subrezent;

Stückzahl: Mtc: 1, Mtt: 1 (n. Gromova 1949).

Ë.spec., Freyburg a.d. U.: (kleine Form = Freyburg b) Würm;

Stückzahl: Mtc: 5, Mtt: 8 (nach Schwarz 1927 u. v. Reichenau 1915).

E. spec. Freyburg a.d. U.: (größere Form = Freyburg a), Würm;

Stückzahl Mtc 2, Mtt 5 (nach Schwarz 1927).

N. B. Im Freyburger Löß kommen offensichtlich zwei verschiedengroße Pferde vor, wie aus dem Diagramm ersichtlich.

E. spec. Solutré, Spätwürm;

Stückzahl: Mtc: 40 (Länge) 7 (Breite), Mtt: 44 (Länge), 4 (Breite)

(aus Gromova 1927 u. n. Viret 1954).

Von allen Materialien ist hier zweifelsohne die größte Einheitlichkeit in Bezug auf Zeitstellung und Klima gegeben. Da in Mitteleuropa im Spätwürm aber mindestens zwei verschiedene Pferde auftreten, ist die taxionomische Einheitlichkeit fraglich. Leider sind in der Literatur nicht Einzel-, sondern nur Grenzwerte für die Metapodien angegeben, eine Entscheidung, ob eine oder zwei Größengrupoen vorhanden sind, kann erst durch eine neue umfassende Bearbeitung erfolgen.

E. spec. Körbisdorf, Riss;

Stückzahl: Mtc 8, Mtt: 14 (nach Schwarz 1927)

Eine gewisse stratigraphische und klimatische Einheitlichkeit der Fundschichten darf vermutet werden. Eine morphologische Analyse der Körbisdorfer Equiden fehlt.

E. mosbachensis v. ReichenuU, Mosbacher Sande, Jüng. Altpleistozän;

Stückzahl: Mtc 9, Mtt 16 (n. Schwarz 1927 u. v. Reichenau 1915). Die Mosbacher Sande bilden weder eine Alters- noch eine oekologische Einheit. Die meisten Pferdereste stammen aber aus dem Hauptlager, so daß eine gewisse Einheitlichkeit angenommen werden darf, was auch durch die morphologischen Befunde gestützt wird (v. ReICHENAU 1915).

E. stenonis Cocн., St. Vallier, Alt. Altpleistozän,

Stückzahl: Mtc: 15, Mtt: 32 (n. VIRET 1954).

Nach den geologischen Verhältnissen der Fundlokalität und nach dem morphologischen Befund darf Einheitlichkeit angenommen werden; E. stenonis wird aber an dieser Fundstelle noch von einem größeren Pferd, E. bressanus VIREt begleitet.

E. „sanmeniensis" T. DE JARD. et PIV., China, Altpleistozän;

Stückzahl: Mtc: 22, Mtt: 29 (aus Gromova 1949 und nach Viret 1954).

Die weite geographische Streuung der Fundorte und die große Spanne des zeitlichen Auftretens von $E$. „sanmeniensis“ läßt von vornherein Uneinheitlichkeit vermuten; dies wird durch Viret (1954) bestätigt. Nach diesem Autor besteht E. sanmeniensis aus einer ver-

1) M. W. ist auch für die Art bzw. Unterart E. gmelini Ant. bisher auch noch kein Typenexemplar festgelegt worden. 
hältnismäßig großen und einer sehr kleinen Form (im Diagramm ist nach den Angaben Viret's das Feld auch für die Mtc aufgegliedert, bei den Mtt sind nur die Maße der großen Form berücksichtigt). Nach den von ZDANsKY ermittelten Zahlen (n. Gromova) scheinen aber Übergänge vorzukommen.
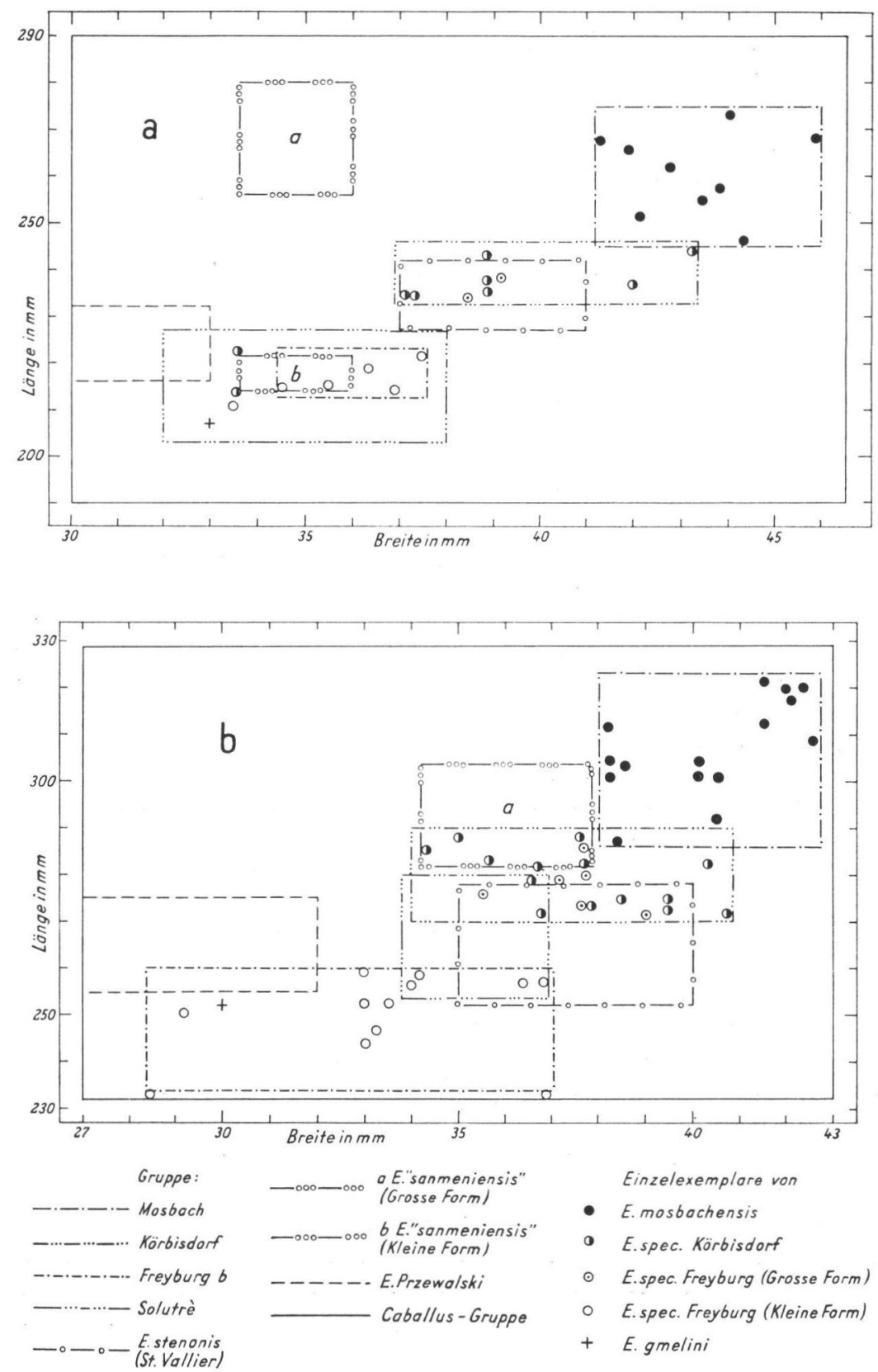

Abb. 2. Verschiedene Typen unter den Pferden der Caballus-Gruppe (einschl. „Allobippus“). a: Metacarpale, b: Metatarsale. 
Das Diagramm 2 erlaubt folgende Feststellung:

1) Die untersuchten west- und mitteleuropäischen Formen gestatten eine Aufgliederung in 4 Größengruppen ("Typen“):

a) Typus Mosbach: Groß- und plumpwüchsig²)

b) Typus Körbisdorf: Mittelgroß und plump

c) Typus E.stenonis, St. Vallier. Wrie Körbisdorf, nur etwas kleiner

d) Typus Freyburg b: Klein und plump. NB. Freyburg a gehört zum Typus Körbisdorf.

2) Bei den Typen $a, b$ und d verhalten sich die Mtc und Mtt in Bezug auf ihre Dimensionierung gleichsinnig, das kleinere Pferd von St. Vallier besitzt dagegen vergleichsweise längere Mtc und kürzere Mtt; es vertritt daher einen grundsätzlich anderen Proportionierungstyp.

3) Die 4 Gruppen bilden eine „allometrische Reihe“, d. h. mit der Größe nimmt auch die relative Plumpheit zu. Merkwürdigerweise entspricht dem nicht die Verteilung der einzelnen Exemplare im zugehörigen Feld. Die Anordnung zeigt im Gegenteil eher das Bild einer negativen Allometrie, d. h. die größeren Exemplare sind zugleich relativ schlanker. Bei den Mtt (abgeschwächt auch bei den Mtc) des Typus Mosbach lassen sich zwei Untergruppen erkennen, jede mit einer merklichen Tendenz zu einem negativ allometrischen Verhalten (Geologische Altersverschiedenheit? Sexuelle Unterschiede?).

4) Sämtliche 4 Typen gehören in die Reihe der „schweren“ Pferde mit kurzen Metapodien.

5) Bei den geologisch ältesten Typen (Mosbach), abgeschwächt auch beim Typus St. Vallier, schwankt die Länge verhältnismäßig stärker (Längeninstabilität), bei den jüngeren Typen Körbisdorf und Freyburg b besonders beim letztgenannten (Mtc!) dagegen die Breite (Breiteninstabilität). Zieht man die große Zahl der Exemplare beim Typus Mosbach und Typus St. Vallier in Betracht, dann ist die Größenschwankungsbreite, ausgedrückt durch die relative Feldgröße überhaupt bei jenen bedeutender als bei den älteren Typen.

6) Das Przewalskipferd hat mittelgroße, aber zugleich schlanke Metapodien. Mtc und Mtt verhalten sich gleichsinnig. Das durch das rezente Wildpferd besetzte Feld ordnet sich $\mathrm{nicht}$ der oben aufgestellten allometrischen Reihe ein, es ist kein plumpes Pferd.

7) Das gleiche gilt von E. „sanmeniensis“, wenigstens was die Großform angeht. Diese ist in ihrer Mtc großwüchsig und zugleich schlank, in ihren Mtt dagegen ist sie nur über mittelgroß und dabei mäßig plump. Mtc und Mtt verhalten sich also ebenso wie bei E. stenonis von St. Vallier $\mathrm{n} \mathrm{ich} \mathrm{t}$ gleichsinnig. Noch ausgeprägter als bei den beiden europäischen Altpleistozäntypen ist die relative Längeninstabilität und die relative Breitenstabilität.

8) Das eine Tarpanexemplar nimmt seinen Platz im Felde des Typus Freyburg b ein.

9) Das Solutrématerial läßt sich nicht den vier ermittelten Typen zuordnen. Nach den Mtc gehört es zum Typus Freyburg b, die Mtt sind aber im Verhältnis zu den Mtc etwas größer und plumper, ihr Feld überdeckt sich in seiner oberen Hälfte mit jenen des Typus Körbisdorf, in seiner unteren dagegen mit jenen des Typus Freyburg b. $\mathrm{Ob}$ tatsächlich sämtliche Metapodien nur e in e $r$ Form angehören, die einen Typus repräsentieren würde, wie er sonst bei den europäischen Pferden nicht auftritt (Un-

2) Ein in der Tabelle bei Schwarz (1927) geführtes Mtt (Mosbacher Sande, Lok. „Hessler“) m. einer größten Länge von $371 \mathrm{~mm}$ und einer Diaphysenbreite von $34,5 \mathrm{~mm}$ weicht dermaßen von den für $E$. mosbachensis gewöhnlichen Dimensionen $a b$, daß es hier unberücksichtigt blieb. Wenn nicht überhaupt ein Druckfehler vorliegt, so ist dieses Stück entweder überhaupt nicht auf E. mosbachensis zu beziehen, was wahrscheinlicher ist, oder es stammt von einem sehr jugendlichen Exemplar. 
gleichsinnigkeit der Proportionen der Mtc und Mtt, aber anders als bei E. stenonis von St. Vallier und E. sanmeniensis, große Form) oder ob ein Formengemisch vorliegt, kann erst entschieden werden, wenn ausreichende, von einer Hand unternommene Messungen durchgeführt werden.

\section{IV}

Die bisher getroffenen Feststellungen und Überlegungen gestatten einen Schritt weiter zu gehen und Diagramme aufzustellen, in welchen sämtliche mir zugänglich gewordenen Werte des europäischen und nordasiatischen nachmindelzeitlichen Materials eingetragen sind, soweit es sich auf Angehörige der Untergattung Caballus bezieht.

Aus den Diagrammen läßt sich nicht nur die Größenposition jedes einzelnen Restes erkennen, sondern es wurde auch versucht, seine chronologische und ökologische Stellung sichtbar zu machen. Daß dabei nur sehr grob verfahren werden konnte, bedarf keiner beconderen Begründung. Als chronologische Kategorien erscheinen: Holstein, Riss, Eem, Würm und „Alter ungewiß, aber zweifelsfrei nachmindelzeitlich", die ökologische Gruppierung erfolgte nach: warm (vollwarmzeitlich), kühl-kalt, vollkaltzeitlich und Temperaturverhältnisse ungewiß (z. T. mit den Varianten „ungeklärt“ und „vermutlich mit Warmeinfluß" (=gemäßigt, Interstadial, wärmere Phasen einer Kaltzeit). Die Zahlenwerte wurden den Arbeiten von Gromova (1949), Schwarz (1927), Wernert (1957), Weiler (1937), Arambourg (1958), v. Reichenau (1915) u. a. entnommen, zum geringsten Teil beruhen sie auch auf eigenen Messungen (Steinheim/Murr, Wunstorf, Aschenstein).

\section{a) West - und Mitteleuropa}

(Diagramme $3 \mathrm{a} \mathrm{u.} \mathrm{b)}$

Ohne den Verhältnissen zu großen Zwang anzutun, kann eine Gruppierung des gesamten Materials nach den oben aufgestellten Typen Mosbach, Körbisdorf und Freyburg b vorgenommen werden. Der altpleistozäne Typus St. Vallier, dessen Zugehörigkeit zur Untergattung Caballus unwahrscheinlich ist, bleibt in diesem Zusammenhang unbeachtet. Schwarz hat in seiner Arbeit ebenfalls unter Benutzung der Metapodien sein Material nach drei Größengruppen geordnet, die den Typen Mosbach, Körbisdorf und Freyburg b entsprechen (1927). Im Grundsatz herrscht also zwischen seiner und unserer Auffassung Übereinstimmung. Nur ein allerdings sehr wesentlicher Unterschied besteht, während SCHwarz in diesen drei Gruppen zugleich drei selbständige Unterarten erblickt, die von ihm als E.caballus robustus Poм., E. caballus plicidens Owen u. E. caballus caballus L. bezeichnet werden, wird hier eine derartig vereinfachende taxionomische Ausdeutung abgelehnt.

1. Der Größentypus Mosbach tritt in der Holstein-Warmzeit (Achenheim, Wunstorf) und in der Eem-Warmzeit auf (Weimar, Burgtonna, Gräfentonna, Phöben), der Klimacharakter der Fundschichten kann als ziemlich gesichert angesehen werden. Dagegen bleibt dieser bei den unten zu nennenden Fundlokalitäten zweifelhaft, da entweder die betreffenden Ablagerungen verschiedene, sich klimatisch nicht entsprechende Altersstufen umfassen (Fontéchevade und Roter Berg) oder der Verdacht auf Vorkommen auf sekundärer Lagerstätte nicht von der Hand zu weisen ist (Mittenwalde, Rixdorfer Horizont!), oder die geologischen und damit klimatischen Verhältnisse nicht klar genug sind (Vieselbach, Heiligenstadt). Sicher ist aber, daß nirgends ein Pferd von der Größe des Typus Mosbach in Gesellschaft einer typischen Kaltfauna auftritt. Weiter läßt sich feststellen, daß in keinem Falle ein würmzeitliches Alter eines großen Pferdes sich beweisen läßt, wenn auch im Falle Heiligenstadt und Mittenwalde die Anwesenheit eines solchen im frühen Würm (Göttweiger Interstadial?) sich andererseits nicht ganz ausschließen läßt. Die Proportionsunterschiede bei der großen warmzeitlichen Form sind bedeutender als beim Typus Mosbach s. str., da neben einer sehr plumpen Form auch eine sehr große, schlanke auftritt (Burgtonna, s. auch S. 107). 


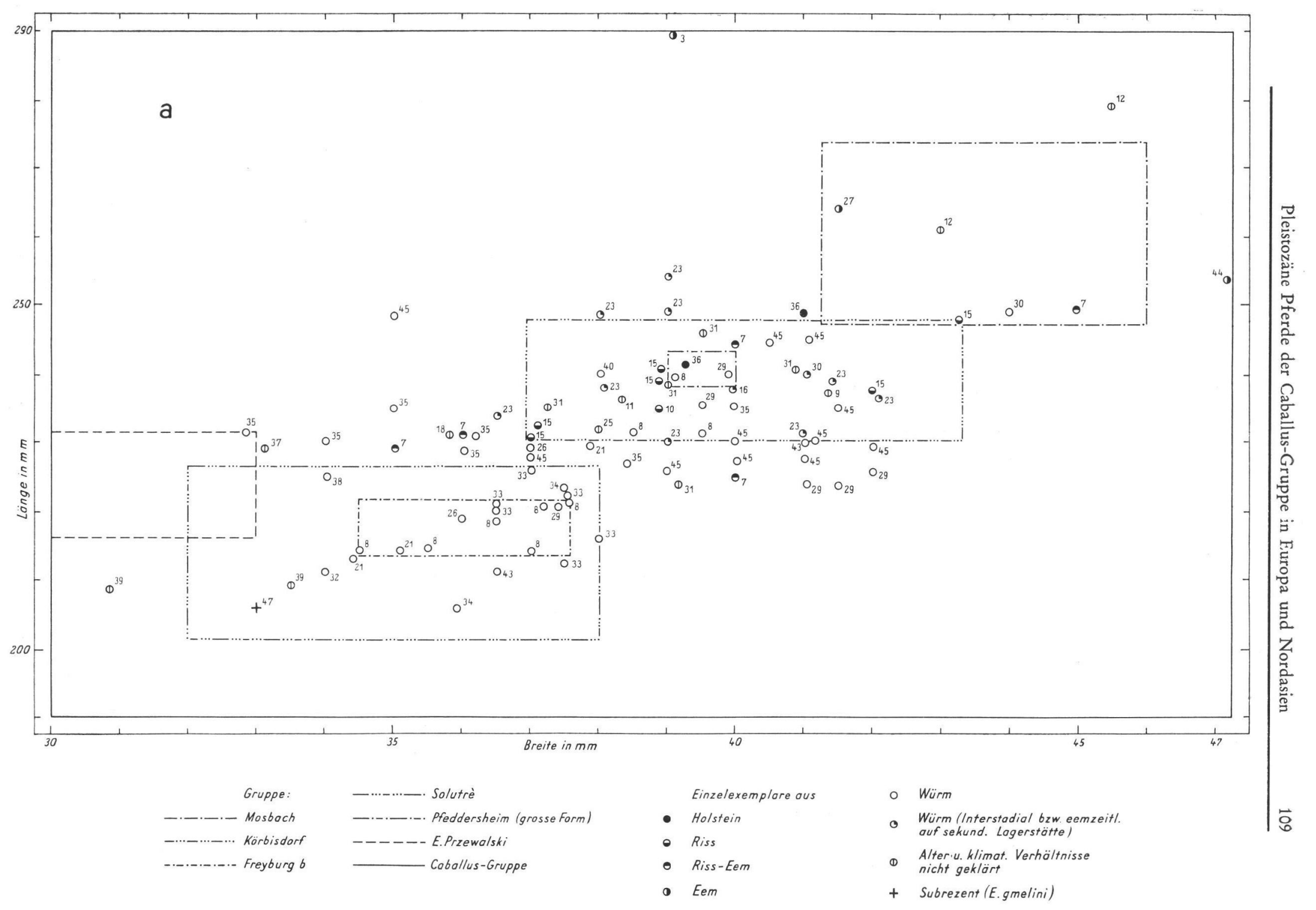




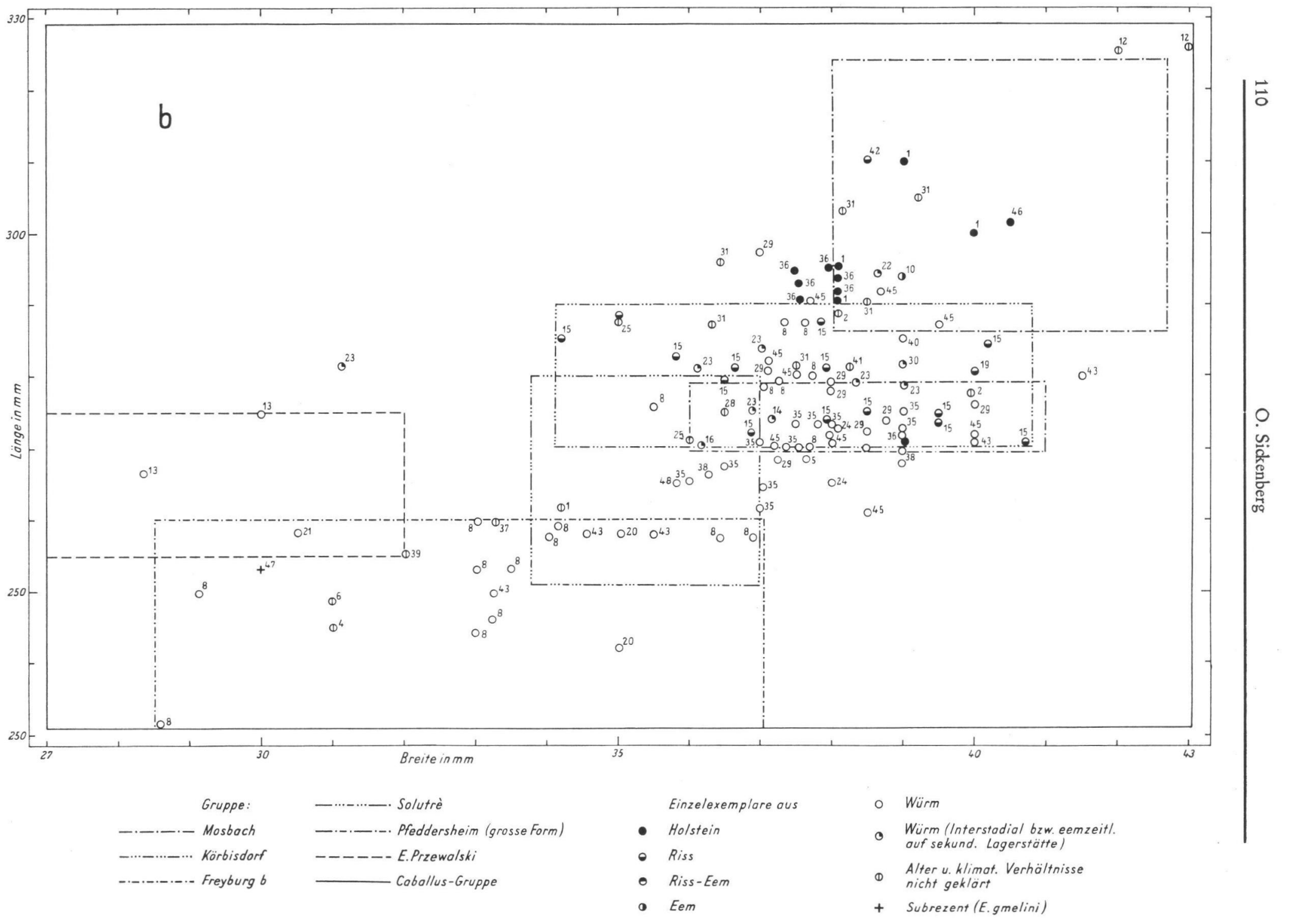


2. Die überwiegende Zahl der nachmindelzeitlichen Pferde zeigt Mittelgröße, die durch den Typus Körbisdorf bestimmte Größenordnung wird aber z. T. nicht unwesentlich über-, vor allem aber häufig unterschritten, was die Längendimension angeht, auch der Breitenspielraum erweist sich als bedeutender; für eine in sich geschlossene Form, etwa im Range einer guten Unterart ist dièse Variabilität entschieden zu groß, auch wenn die bedeutende zeitliche Streuung in Rechnung gestellt wird. Der Typus Körbisdorf stellt offenbar ein Formengemisch dar, über dessen Natur noch zu sprechen sein wird.

Die Mehrzahl der Reste läßt sich ohne weiteres dem Körbisdorfer Typus s. str. zuordnen. Es sind jene der Fundlokalitäten Westeregeln z. T., Quedlingburg (große Form), Freyburg (gr. F.), sämtliche Lokalitäten des Rixdorfer Horizontes mit Ausnahme Mittenwalde (Niederlehme, Rixdorf, Körbiskrug, Kreuzberg, Königswusterhausen), Görsdorf, Barley, Halbe, Roter Berg (kleine F.), Unkelstein (Type von E. germanicus), Pfeddersheim (gr. F.), Fontéchevade (z. T.), Steeden (z. T.), Oreston Cave, Vogelherd (gr. F.), Pössneck, Lengefeld, Veltheim, Ofnet (z. T.). Dem Alter nach gehört das Material entweder ins Riß oder Würm, vermutlich mit Ausnahme des einen Steinheimer Exemplares. Bei keinem Stiick ist eemzeitliches Alter sicher (Fontéchevade?, Roter Berg?). Für einen größeren Teil sind kältere Klimabedingungen während der Bildungszeit der Fundschichten nachweisbar, die Temperaturverhältnisse einer Vollwarmzeit umgekehrt höchstens für Fontéchevade, vielleicht auch für Steinheim anzunehmen.

Größer als es dem Zentralbereich des Typus entspricht, sind die Exemplare von Steinheim (mit einer Ausnahme), Achenheim, Roter Berg z. T., Quedlinburg (?). Sie könnten gegebenenfalls auch für kleinwüchsige Vertreter des Mosbacher Typus angesehen werden. Ein höheres als würmzeitliches Alter ist bei Steinheim und Achenheim sicher, bei „Roter Berg“ möglich. Die Temperaturen sind warm bis gemäßigt. Altersmäßig und ökologisch paßt das große Quedlinburger Exemplar jedoch nicht in diese Gruppe. Kleiner sind: Quedlinburg z. T., Westeregeln z. T., Steeden z. T., Roter Berg z. T., Vogelherd z. T., Fontéchevade z. T., Thiede, Ofnet z. T., Datteln, Aschenstein. Keine Fundstelle ist älter als das Riß und nur Fontéchevade und vielleicht auch Roter Berg älter als Würm. Sieht man von den beiden letztgenannten Fundplätzen ab, können kaltzeitliche Klimazustände vorausgesetzt werden.

Schlanker sind: Steeden z. T., Solutrè (z. T.?), Streckau, Lauchstedt, Fontéchevade z. T., Westeregeln (subfossil?). In den Proportionen nähert sich diese Subvariante den Proportionen des Przewalski-Pferdes. Möglicherweise handelt es sich auch um beson-

Abb. 3 (vor- u. nebenstehend). Nachmindelzeitliche Pferde in Mittel- und Westeuropa. a: Metacarpale, b: Metatarsale. Die Bezifferung kennzeichnet folgende Fundorte:

$\begin{array}{llll}1 \text { Achenheim } & 13 \text { Kalla } & 25 \text { Oreston Cave } & 37 \text { Streckau } \\ 2 \text { Barley } & 14 \text { Königswusterhausen } & 26 \text { Pfeddersheim } & 38 \text { Thiede } \\ 3 \text { Burgtonna } & 15 \text { Körbisdorf } & 27 \text { Phoeben } & 39 \text { Kösten b. Türmitz } \\ 4 \text { Koburg } & 16 \text { Körbiskrug } & 28 \text { Poessneck } & 40 \text { Unkelstein } \\ 5 \text { Datteln } & 17 \text { Kreuzberg } & 29 \text { Quedlinburg } & 41 \text { Veltheim } \\ 6 \text { Dörstewitz } & 18 \text { Lauchstedt } & 30 \text { Rixdorf } & 42 \text { Vieselbach } \\ 7 \text { Fontéchevade } & 19 \text { Lengefeld } & 31 \text { Roter Berg } & 43 \text { Vogelherd } \\ 8 \text { Freyburg u.d.U. } & 20 \text { Lingolsheim } & 32 \text { Schussenquelle } & 44 \text { Weimar } \\ 9 \text { Görsdorf } & 21 \text { Mainz-Kastell } & 33 \text { Solutrè } & 45 \text { Westeregeln } \\ 10 \text { Gräfentonna } & 22 \text { Mittenwalde } & 34 \text { Sossenheim } & 46 \text { Wunstorf } \\ 11 \text { Halbe } & 23 \text { Niederlehme } & 35 \text { Steeden } & 47 \text { E.gmelini } \\ 12 \text { Heiligenstadt } & 24 \text { Ofnet } & 36 \text { Steinheim/Murr } & 48 \text { Aschenstein }\end{array}$


ders große Vertreter des Typus Freyburg b, jedoch ist dies nicht sehr wahrscheinlich. Sieht man wieder von Fontéchevade ab, ist würmzeitliches Alter sicher oder vermutbar. Das Gesamtbild erlaubt folgende Feststellungen:

Innerhalb des Formengemisches Typus Körbisdorf sind einerseits die geologisch älteren (Steinheim, Achenheim, Körbisdorf), andererseits die ökologisch „wärmegetönten" Formen (z. B. Roter Berg z. T., Rixdorfer Horizont) mit einiger Deutlichkeit durchschnittlich größer als die jüngeren und zugleich unter ungünstigeren Klimabedingungen lebenden (über die Pferdereste von Fontéchevade s. S. 120).

3. Da zwischen den einzelnen Varianten des Typus keine scharfen Grenzen bestehen, Übergänge also vorhanden sind, besonders zwischen dem Zentraltypus und seinen Kleinvarianten, bietet sich die Annahme, es hätte in den einzelnen unabhängig verlaufenden Stammlinien eine fortschreitende Größenabnahme vom Holstein bis zum Endwürm stattgefunden, als nicht unwahrscheinlich an, da ja die geologisch jüngeren Vertreter des Typus im allgemeinen kleiner sind. Der Längenschwund war aber allem Anschein nach nicht von einer Verringerung der Breite im Sinne einer positiven Allometrie begleitet, mit anderen Worten, die kleineren Pferde waren verhältnismäßig plumper.

4. Zu dem durch das kleinere Freyburger Pferd (Freyburg b) gegebenen Typus zählen ausnahmslos Exemplare, deren würmzeitliches Alter entweder beweisbar ist oder wahrscheinlich gemacht werden kann. Allerdings besteht bei dem einen oder anderen Stück gerade dieses Tvpus aus älteren Aufsammlungen die Möglichkeit, daß altholozänen Wild- oder gar Hauspferdresten ein höheres Alter zugeschrieben wurde, da Vermengung schon am Fundplatz (gestörte Höhlenablagerungen!) oder durch unvollkommene Grabungstechnik eingetreten ist. Der Verdacht auf interstadiales Alter und dementsprechendem Klima besteht in keinem einzigen Falle. Vielfach läßt sich sogar eine Einstufung in den Zeitabschnitt Würm II u. III vornehmen. Im Falle des Fundkomplexes Vogelherd, Achenheim, Pfeddersheim, Quedlinburg und wie schon erwähnt, Freyburg selbst, wird das kleinwüchsige Pferd des Typus Freyburg b von einem größeren Pferd begleitet, das dem Typus Körbisdorf angehört und das offenbar zumindest teilweise gleichzeitig mit ihm zusammen gelebt hat. $\mathrm{Zu}$ ähnlichen Feststellungen sind bereits andere Autoren gelangt, wobei sie sich nicht nur auf metapodiales Material beziehen (z. B. LeHMANN 1953, LundHolm 1949). Weitere Vorkommen sind: Lingolsheim, Dörstewitz, Koburg, Kösten b. Türmitz, Mainz-Kastell, Sossenheim, Schussenquelle, Streckau. Unklar ist, wie bereits erwähnt, die Stellung des Solutrèmaterials, das nach den Ausmaßen der Mtc eindeutig zum Typus Freyburg b gehört, in Bezug auf die Dimensionen der Mtt sich dagegen stark dem Typus Körbisdorf nähert. Auch die Zugehörigkeit der Kleinform von Fontéchevade, des Lauchstädter Pferdes und eines Teiles der Steedener und Thieder Materialien muß offen bleiben. Von Wichtigkeit ist aber, daß wenigstens nach dem einen vermessenen Exemplar zu schließen, der Tarpan zum Typus Freyburg b zählt. Keine Verbindung kann aber zum Przewalski-Pferd hergestellt werden, auf dessen besondere Stellung im Rahmen der gesamten Untergattung bereits hingewiesen wurde. Das Fehlen eines Pferdes von den Extremitätenproportionen des E. Przewalski unter den pleistozänen Equiden Europas kann daher als erwiesen gelten, wenn man von dem durch zwei Mtt belegten Pferd des Fundplatzes Kalla bei Biebrich absieht. Die Stücke sollen aber oberflächennah (?) in einem Sandlöß gefunden worden sein, der Mosbacher Sande überlagert. Die Möglichkeit besteht also immerhin, daß es sich um Fundgut aus einer prähistorischen Station handelt, was ich stark vermuten möchte. Sind es aber tatsächlich Reste eines pleistozänen Wildpferdes, wäre dies als Hinweis aufzufassen, daß im Würm Mitteleuropas neben einem genügend belegtem tarpanoiden Pferd auch eine dem heutigen mongolischen Wildpferd entsprechende Form als Seltenheit vorkam. 
b) Osteuropa und Nordasien

(Diagramme $4 \mathrm{a}$ u. b)

Anzahl der für die Aufstellung der Diagramme herangezogenen Exemplare von folgenden Lokalitäten:

Mtc: Jana 17

Mtt: Unga 3, Jana 16, Stary Kodak 3, Kubiekow 5, Kotelny?

Die Anzahl der Stücke der anderen Lokalitäten läßt sich unmittelbar aus den Diagrammen ablesen.

Die Mehrzahl der Funde ist, ähnlich wie in Mittel- und Westeuropa, geologisch jung (Würm II u. III). Sicher älter sind ein Teil der nordsibirischen Stiicke, die aus Schichten stammen, die nach Fauna und Flora nur in einem Vollinterglazial entstanden sein können (wahrscheinlich Eem, vielleicht auch Holstein z. T.). Ins Riß sind möglicherweise die Fundschichten der Pferde von Mysy (E. caballus missi PAvL.) und Tungur (E. caballus chosaricus Groм.) einzustufen. Weitere Einzelheiten können den Angaben des Anhanges entnommen werden; auch auf die Schwierigkeiten, die quartären Bildungen des osteuropäisch-nordasiatischen Raumes in richtige zeitliche Beziehungen $\mathrm{zu}$ jenen des übrigen Europas zu bringen, sei hier hingewiesen. Besonderer Betonung bedarf es nicht, daß eine zutreffende Beurteilung der klimatischen Verhältnisse, die während der einzelnen Phasen des Pleistozäns in den verschiedenen Teilräumen dieses riesigen Gebietes geherrscht haben, noch bedeutend schwieriger ist, als für die vergleichsweise winzige Fläche W'est- und Mitteleuropas. Die kontinentale Größe des Raumes, der in keinem Zeitabschnitt des Pleistozäns auch nur annähernd gleichgeartete Landschaften umschloß, ließe erwarten, daß in diesen eine erheblich größere Anzahl verschieden proportionierter Pferde als im Westen gelebt hätten. Um so überraschender ist daher, daß sich die allerdings spärlichen Funde, die bisher gemacht wurden, leicht in die für die westlichen Gebiete aufgestellten Größenkategorien einreihen lassen. Aber nicht nur diese Einreihung vollzieht sich unschwierig, allem Anschein war auch das stammesgeschichtliche Geschehen sehr ähnlich, in dem nämlich auch im Osten Europas und Norden Asiens offenbar die geologisch älteren Formen die größeren, die kleineren zugleich die jüngeren sind. Auch das Fehlen von großen Pferden in kalttemperierten Gebieten ist wahrscheinlich, wenn auch natürlich nicht schlüssig zu beweisen. Im einzelnen ergibt sich folgendes Bild:

1. Große Pferde vom Typus Mosbach sind nur aus dem hohen Norden belegt: Tavda und Jana (z. T.). Da aus Schichten ü b e r dem Bodeneis (=Würm) bis jetzt nur kleine Pferde bekannt wurden (Taimir-Halbinsel, Kotelny), kann mit einiger Sicherheit für die großen Formen vollwarmzeitliches Alter angenommen werden (Eem?, Holstein ?).

2. Die Mehrzahl der Pferde gehört auch hier dem Typus Körbisdorf an. Dieser Typus wird als Formengemenge aufzufassen sein, das auch die als E. caballus missi PAvL. und E. caballus chosaricus Grom. beschriebenen Pferde umfaßt. In der oberen Hälfte des Feldes liegen die Werte jener Pferde, für die ein höheres Alter als Würm II u. III bzw. eine wärmegetönte Fazies ihrer Umgebung anzunehmen ist: Tavda, Ilskaja, Tungur, Scholchowo, in der unteren Hälfte befinden sich die geologisch jüngeren Formen bzw. solche, die unter kühlen bis kalten Temperaturbedingungen lebten: Mysy, Südural (z. T.), Borchievo, Kostenki, Novgorod-Severski, Koch-Koba, Adji-Koba, Stary Kodak. Da die Fundschichten von Kubiekowa eine Zeitspanne umfassen, die vom Holstein (?) bis ins Spätwürm reichen soll, überrascht es nicht, daß die Werte weit gestreut sind und ein übermittelgroßes Pferd des Typus Körbisdorf und eines des Typus Freyburg b anzeigen.

3. Für die Kleinpferde des Typus Freyburg b ist ein spätwürmzeitliches Alter entweder erwiesen oder zu vermuten (Tschikoi, Jana z. T., Anabar, Kubiekowa z. T., Vierkholenskaja gora). Das Süduralpferd lebte anscheinend unter nicht ungünstigen Klima-

8 Eiszeit und Gegenwart 

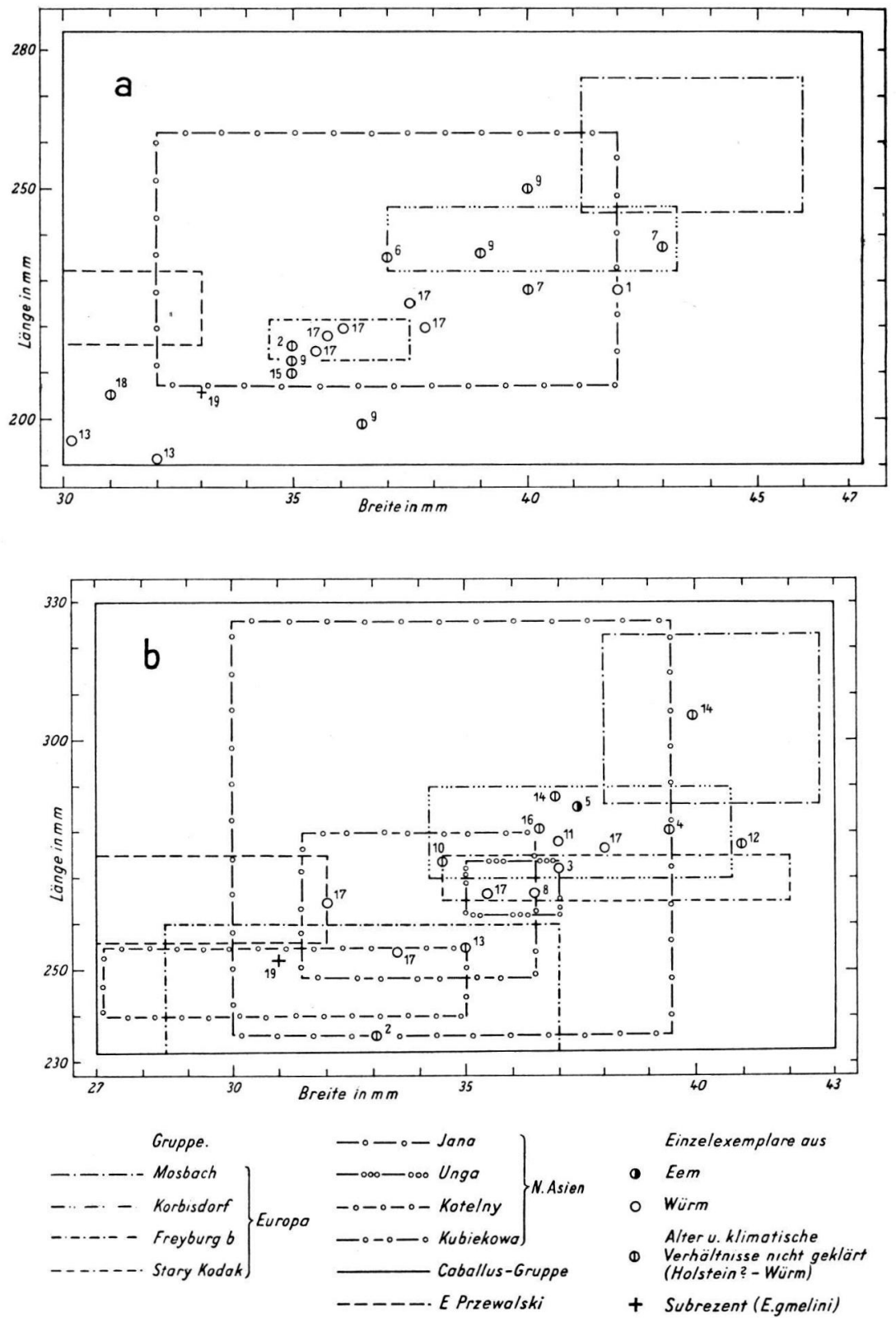

Abb. 4. Nachmindelzeitliche Pferde in Osteuropa und Nordasien. a: Metarcarpale, b: Metatarsale. Die Bezifferung bezeichnet folgende Fundorte:

1 Adji Koba

2 Anabar

3 Borchevo II

4 Bugatsch

5 Ilskaia

6 Koch Koba
7 Kodak (Stary Kodak)

8 Kostenki I

9 Kubiekova

10 Mysy

11 Novgorod-Severski
12 Scholokova

13 Taimir

14 Tavda

15 Tschikoi

16 Tungur

17 Ural (Südural: Bura- novka, Griebnievaia, Kotchari II, Kliutschewa, OrlovskyHöhle, Ust-Katav II) 18 Vierkholenskaja gora 19 E. gmelini 
bedingungen, die nordsibirischen Tiere (Taimir, Anabar, Kubiekowa, Jana z. T.) sind die kleinsten bis jetzt bekanntgewordenen pleistozänen (und rezenten) Angehörigen der Untergattung überhaupt. Ebenso wie die europäischen, zeigen auch die asiatischen Kleinpferde in ihren Proportionen den Tarpan- und nicht den Przewalski-Typus, auch in Asien ist dieser unter dem fossilen Material nicht vertreten. $\mathrm{Zu}$ ihm könnte allenfalls nur das eine Exemplar von Vierkholenskaja gora (Spätwürm?, Holozän?) passen.

\section{$\mathbf{V}$}

Um die Grundlage für die zu treffenden Folgerungen zu verbreitern, empfiehlt sich, zum Vergleich noch einen weiteren Formenkreis heranzuziehen. Am geeignetsten ist für diesen Zweck die Untergattung Amerhippus in ihren südamerikanischen Vertretern (Diagramme $5 \mathrm{a}$ u. b). Über diese Gruppe liegt eine neuere Beschreibung vor, die sehr geeignet ist, die verfolgten Absichten zu unterstützen (HofstetTER 1952). Die südamerikanischen Arten von Amerbippus sind A.neogaeus Lund, A. Santae-Elenae SpILlm., A. Martinei Spillm., A.insulatus C. Ameghino, A. Andium Wagner-Branco, A.curvidens Owen; die letztgenannte ist allerdings nach HoFstetrer als taxionomische Einheit „unrein“, da sie ein Formengemenge darstellt. Während die beiden erstgenannten Arten Bewohner von tropischen Flachländereien waren, lebten A. insulatus (Altpleistozän), A. Martinei (Mittelpleistozän) und A. Andium (Jungpleistozän) in den Hochanden. Der Lebensraum von $A$. „curvidens" schließlich waren die Ebenen, die sich im Süden an das Verbreitungsgebiet von $A$. neogaeus anschlossen.

Aus einem Vergleich der betreffenden Diagramme (2, 3, 4 u. 5) ergibt sich eine nahe$\mathrm{zu}$ vollständige Übereinstimmung, die fast verblüffend genannt werden kann. Dies wird nur unter der Annahme verständlicher, die Entwicklung beider Equidengruppen sei von den gleichen Formungskräften gesteuert. Wie bei den nachmindelzeitlichen Formen von Caballus lassen sich drei verschiedene Größen unterscheiden, wobei die durch die vorherrschende Mittelgröße gekennzeichnete Gruppe auch hier ein Formengemenge ist und verschiedene Arten einschließt. Die Zunahme der Länge und Breite der Metapodien von Größenstufe zu Größenstufe folgt einer positiven Allometrie, aber auch bei Amerhippus sind Tendenzen zu verzeichnen, innerhalb einer Art die positive in eine negative Allometrie umzukehren. Wie dies auch bei Caballus die Regel ist, besitzen die Mtc und Mtt innerhalb jeder Form die gleichen oder sehr ähnlichen Proportionsverhältnisse. Im ganzen gesehen sind die jeweils arteigenen Proportionen bei den Mtt gefestigter als bei den Mtc. Unter den andinen Formen ist wieder die geologisch jüngste, A. Andium, die kleinste. Eine Beziehung zu klimatischen Faktoren scheint nur bei der Großform (A. neogaeus, tropisches Flachland) und bei den Kleinformen (A. Andium, andines Hochland) klar erkennbar. Undeutlicher verhält sich in dieser Beziehung die Mittelgruppe, obwohl sich auch hier feststellen läßt, daß unter ihren Angehörigen A. Santae-Elenae als Bewohner tropischer Warmgebiete größenmäßig die Spitze hält. In Bezug auf die Plumpheit der Metapodien (Länge : Breite) bestehen zwischen den größeren und kleineren Arten keine ersichtlichen Unterschiede (Allometrie!). Auch A. „curvidens" bildet keine Ausnahme, obwohl von den Angehörigen dieses Formgemenges noch am ehesten ein Leben in offeneren, ebenen Landschaften vorausgesetzt werden darf, wenn nicht wenigstens während längerer Abschnitte des Pleistozäns auch diese Gebiete waldreicher als in der Gegenwart waren.

\section{VI}

Auf Grund der bisherigen Feststellungen kann der Versuch unternommen werden, das Bezugssystem Größenentwicklung und erdgeschichtliches Geschehen, deutend zu untersuchen. Auf teils ähnliche, teils auch stark abweichende Gedankengänge in der bisherigen Literatur (Gromova 1949, Lundholm 1949, Höfstetter 1952, Lehmann 1954, Mohr 

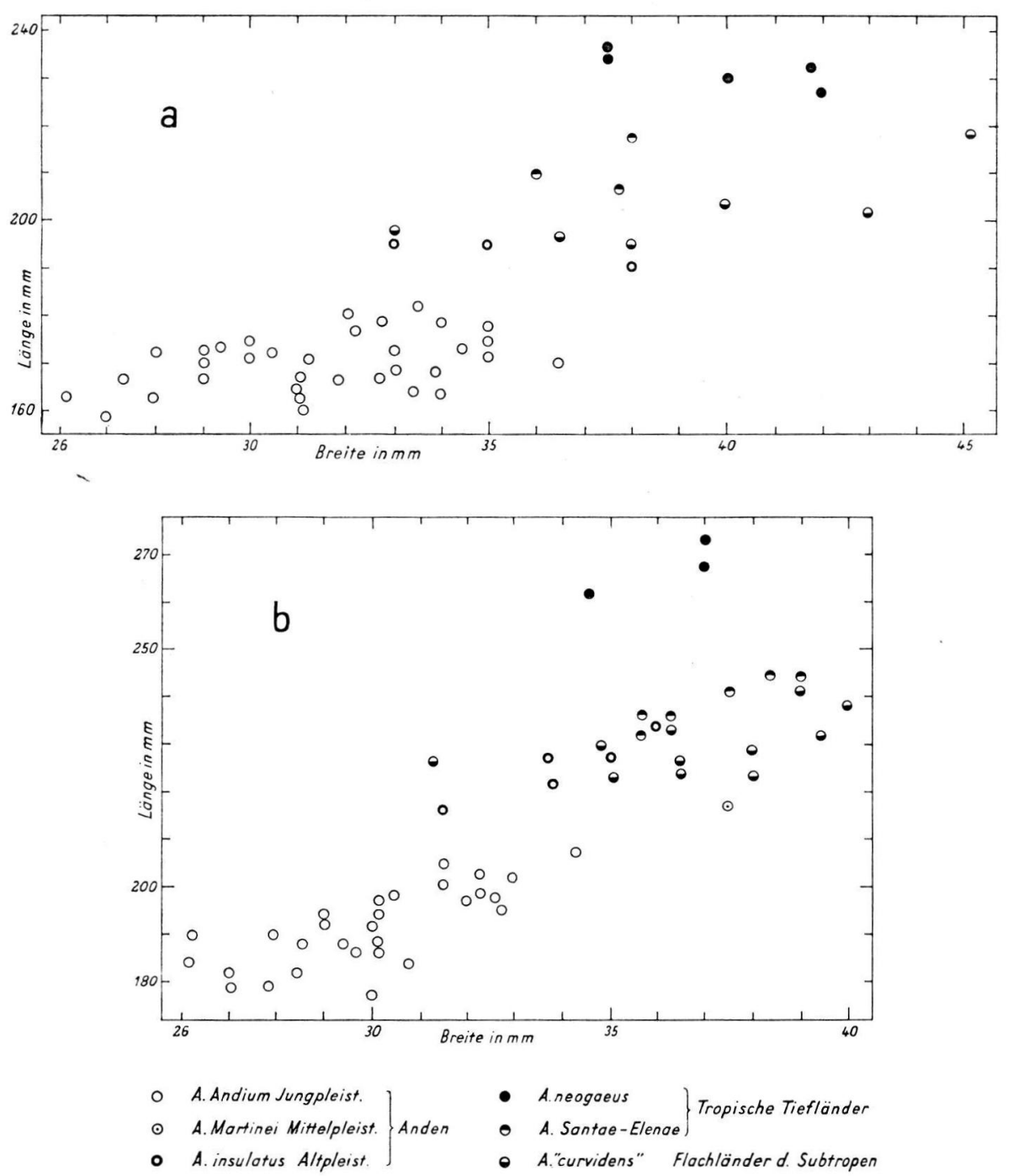

Abb. 5. Südamerikanische Arten der Gattung Amerhippus. a: Metacarpale, b: Metatarsale.

1958, Dietrich 1950, Stehlin 1933, Herre 1938 usw.) sei verwiesen, eine ins einzelne gehende Auseinandersetzung kann in diesem Rahmen aber nicht erfolgen. Vorausgeschickt sei, daß das Phänomen der stammesgeschichtlichen Größenzunahme und -abnahme äußerst vielschichtig ist. Zahlreiche Faktoren sind beteiligt: Ernährung, Klima, Gelände, Fortbewegungsart, Weite und Enge des Lebensraumes (Isolation!), Evolutionsgeschehen (Radiation, Aussterben), Bastardierung, Inzucht (Isolation), vielleicht sogar Großschwankungen der kosmischen Strahlung usw. Bei einer Rechnung mit so vielen Unbekannten haftet jeder Deutung eines bestimmten stammesgeschichtlichen Geschehens Unsicherheit und Unvollkommenheit an.

Innerhalb der Untergattung Caballus sind im jüngeren Altquartär Europas bisher nur große Pferde vom Typus Mosbach bekannt geworden. Kleinpferde fehlen offensichtlich. Umgekehrt fehlen jene zumindest seit dem späteren Würm in Europa und Asien. Der 
Körbisdorfer Typus tritt in seiner Großvariante (E. steinbeimensis) zum ersten Mal zwischen Mindel- und Rißkaltzeit in Erscheinung. Ihm gehören die Mehrzabl der riß- und frühwürmzeitlichen Pferde sowohl in Europa als auch in Nordasien an. Kleinpferde vom Typus Freyburg b fehlen bisher mit geologisch gesicherten Belegen, die älter als das spätere Würm sind. Zunächst scheinen diese Tatsachen dafür zu sprechen, daß in allen Stammeslinien, die man sich vielleicht als engbenachbart vorzustellen hätte, eine gleichmäßige Größenabnahme stattgefunden hätte, wofür vor allem die nicht zu übersehende, gewissermaßen stetige Größenabnahme innerhalb des Typus Körbisdorf vom Beginn des Riß an bis zum Endwürm bzw. Altholozän spricht (vgl. auch Amerhippus). Dieser Größenschwund kann aber, besteht diese Annahme zu recht, nur von einem Teil der Stämme mitgemacht worden sein, denn Großpferde vom Mosbacher Typus fehlen in Europa und offenbar selbst in Nordasien noch im Eem, ja vielleicht sogar noch im frühen Würm, zumindest in den südlichen Gebieten, nicht. Ebenso vergesellschaftet sich der Körbisdorfer Typus mit dem Freyburger b im Würm und Altholozän. Ein Teil der durch Mittelgröße gekennzeichneten Populationen unterlag also ebenfalls nicht der Verzwergung. Es ist mithin auch die Annahme, bereits im Prämindel hätte es Vertreter aller drei Größenkategorien, nur nicht die gleichen Regionen bewohnend, gegeben, vorläufig nicht widerlegbar, ja nicht einmal ganz unwahrscheinlich. Sie wären dann nur etappenweise ausgestorben, wobei die Großpferde die ersten Opfer waren. Das wirkliche Geschehen wird sich aber in der Form eines "sowohl - als auch" vollzogen haben, indem neben großen auch kleinere Pferde, aber wohl nicht Zwerge, schon frühzeitig vorhanden waren, eine echte Größenabnahme wenigstens in bestimmten Zweigen aber zweifellos stattgefunden hat. In diesem Zusammenhang sei daran erinnert, daß die Pferde ganz allgemein leicht und rasch zur Verzwergung neigen („Ponys“, Inselpferde, vgl. Lundholm 1948). Wenn die verschiedene Größe durch innere Faktoren allein bestimmt wäre, würden alle Überlegungen, inwieweit Umweltverhältnisse steuernd oder sogar verursachend eingegriffen hätten, müßig sein. Großpferde treten aber in Mittel- und Westeuropa sowie in Nordasien allem Anschein nach nur in den wärmeren Klimaphasen auf (Cromer, Holstein, Eem), Kleinstpferde scheinen in ihrem Auftreten an ausgesprochene Kaltzeiten gebunden zu sein. Die kleinsten, bisher überhaupt bekanntgewordenen Pferde lebten in Nordsibirien an der Wende PleistozänHolozän. Dem könnte entgegengehalten werden, daß im Altquartär sehr verschieden große Pferde lebten, wie vermutet wird, wenigstens zum Teil zu gleicher Zeit (VIrET 1954). Zu wenig ist aber bisher über das klimatische Geschehen, besonders im älteren Altquartär bekannt, zu unsicher ist dessen Chronologie und zu unklar die taxionomische Stellung der allem Anschein nach recht zahlreichen Formen (Caballus div. spec. ?, Allohippus div. spec., Asinus spec.?) um die für die Caballus-Gruppe gemachten Feststellungen zu entwerten. Auf Grund allgemeiner Erwägungen darf überdies für die altquartären Pferde insgesamt cin ökologisch größerer Spielraum (Eurythermie!) anzunehmen sein. Erst im späteren Pleistozän werden sie, wenigstens teilweise, stenotherm geworden sein. Die mittelgroßen Formen dagegen scheinen, wie auch nicht anders zu erwarten, in ihren ökologischen $\mathrm{Ab}-$ hängigkeiten unbestimmter. Bestehen solche Beziehungen zur Temperatur tatsächlich, so folgt daraus, daß für die Pferde entgegen der Ansicht Gromovi's (1949) nicht die BerGMANN'sche Regel gilt. Auch bei der Untergattung Amerhippus bewohnten die größeren Arten wärmere Gebiete. Bei anderen Pflanzenfressern können dafür gleichfalls Beispiele gefunden werden (Unterarten und Rassen der Gattung Rangifer, Elefanten der Gattung Mammonteus). Auf der anderen Seite darf nicht übersehen werden, daß jene Pferde der Gegenwart, die Bewohner heißtemperierter Regionen sind, samt und sonders, verglichen mit den Formen der Untergattung Caballus kleiner sind, die weiter nördlich lebenden aber etwas größer. Diese Verbreitung der rezenten und pleistozänen Equiden nach ihrer Größe legt den Gedanken nahe, daß gemäßigte Klimabedingungen eine bedeutende Körpergröße begünstigen, mit anderen Worten, die Equiden folgen in der geographischen An- 
ordnung zunächst der BergmanN'schen Regel; von einem gewissen kritischen Punkte an schlägt die Größenentwicklung aber in ihr Gegenteil um, da dann offenbar in den betreffenden Populationen kleinere Tiere durch Auslese bevorzugt werden (Nahrung, Nahrungsaufnahme, Untergrund?). Größenabnahme ist ebenfalls, wie ihr Gegenteil, ein entwicklungsgeschichtlicher Vorgang. Sie in jedem Fall nur als degenerativen Prozeß anzusehen, ist unzulässig. Die Kleinpferde des Jungpleistozäns etwa nur als arktische Kümmerformen anzusprechen, ist daher abwegig. Ist die einmal erreichte Größe aber durch Abnahme der Variabilität und einen damit verbundenen Plastizitätsschwund (Stenothermie!) nach Abklingen der Radiationsphase erblich fixiert worden, dann können die betreffenden Formen eintretenden 'Temperaturänderungen nur mit Arealverschiebungen begegnen; ist dies aus äußeren oder inneren Gründen nicht möglich, so wird das Aussterben unvermeidlich (Großpferde im Laufe des Würm, Kleinpferde in der Nacheiszeit, Tarpan!), wobei die Großformen allem Anschein nach zuerst gefährdet sind. Die Aussichten für das Überleben würden für einen derartigen Stamm nur dort gegeben sein, wo eine Änderung der Umwelt nicht oder nur in geringerem Maße stattfand. Nur in solchen Räumen konnten sich die Equiden bis in die Gegenwart erhalten, während sie an der klimatischen Kampffront ausstarben. Das Schicksal auch anderer Säugergruppen im pleistozänen Eurasien dürfte sich ähnlich gestaltet haben, am vollkommensten scheint die Entsprechung bei den Proboscidiern zu sein: Höhepunkt der Größenentwicklung bei Archidiskodon, Palaeoloxodon und Mammonteus im jüngeren Altquartär, Herausbildung kleinerer Rassen bei $M$. trogontherii bzw. M. primigenius im Norden, Persistieren der Großformen als Konservativstämme im Süden ( $P$.antiquus, M.trogontherii) und Ausweitung ihres Areals in den klimatisch günstigeren Phasen, Aussterben im Laufe des Würms auch in Südeuropa, Weiterleben der nördlichen kleinen Primigeniusrassen bis ins beginnende Postglazial, Erleben der Gegenwart nur in den klimatisch stabilen Räumen Indiens und Afrikas (Euelephas, Loxodonta).

Ein anderes Problem stellt sich mit der Frage der Bedingtheit der Proportionsverhältnisse, also von Schlankheit und Plumpheit der Metapodien bei den verschiedenen Formen ("leichte“ und „schwere“ Pferde). Der Grad der Plumpheit wird durch das Verhältnis Länge : Breite bestimmt („Schlankheitsindex“). Nach den Untersuchungen Hofstettek's (1952) sind außerdem bei den "schweren“ Equiden die Metapodien, verglichen mit den anderen Gliedmaßenabschnitten verkürzt, mit anderen Worten, die Plumpheit wird vor allem durch die relative Kürze von Mittelhand und -fuß hervorgerufen. Wie aber HoFSTETTER betont, greift die Verkürzung (bei etwa gleichbleibender Stärke) auch auf die proximalen Gliedmaßenteile über. In der Literatur wird überwiegend die Auffassung vertreten, in den Großpferden des Pleistozäns seien Wald-, in den mittelgroßen und besonders den kleinen hingegen Tundrabewohner zu sehen. Sie stützt sich dabei auf die Bindung an warmzeitliche Phasen auf der einen, an kaltzeitliche auf der anderen Seite. Die Waldpferde sollen dabei besonders „schwer“, d. h. plump sein. Bei den pleistozänen Pferden der Caballus-Gruppe - ob groß, ob klein - gibt es im wesentlichen aber keine, die schlankwüchsiger oder plumper sind als andere. Sie waren ausnahmslos schwer und hatten verkürzte Metapodien. Man könnte höchstens die da und dort erkennbaren Ansätze zu einer negativen Allometrie innerhalb eines Typus bzw. einer Population als Ausdruck dafür werten, daß der Verkürzungsvorgang als solcher nicht abgeschlossen war.

Extremitäten mit verkürzten Metapodien besitzen so gut wie sämtliche Pleistozänformen, ausgeprägter die Angehörigen der Caballus-Gruppe, undeutlicher und abgeschwächt die große Form des E. sanmeniensis und vermutlich auch die Großformen des Allohippus-Kreises. Schlankwüchsig hingegen sind die Esel, Halbesel und Zebras, aber auch das Przewalski-Pferd ist verhältnismäßig schlank. Plump sind hingegen wieder die südamerikanischen Vertreter von Amerhippus. Ganz allgemein ist die Schlankheit der Extremitäten ein Kennzeichen leichter, rascher und nachhaltiger Beweglichkeit, während 
unter den schwergebauten, d. h. mit verhältnismäßig plumpen, kurzen Metapodien ausgestatteten Formen flinke und ausdauernde Läufer schwerlich zu finden sind. Auf die Pferde angewandt möchte man daher in den plumpen Ausprägungen des Typus insgesamt schlechtere Läufer erblicken. Stärkere oder geringere Beweglichkeit nach Tempo und Ausdauer geht entweder mit einem bestimmten psychischen Verhalten (Kalt- und Warmblut!) Hand in Hand oder steht in Verbindung mit der Art des Lebensraumes. In der Rege] werden ja Verhalten und Biotop sich entsprechen, wobei offenbleiben muß, was Ursache und was Folge ist. Möglicherweise wird auch die Temperatur, besonders wenn sie sich extremen Werten nähert, von Einfluß auf die Proportionen sein. Bei den Pferden scheint dies allerdings nicht der Fall zu sein, denn eben dieselben Proportionen können sowohl bei den Warmformen, wie bei den Kaltformen der Untergattung Caballus und Amerhippus festgestellt werden. Auch die Verschiedenartigkeit der Nahrung als mitbeteiligt anzusehen, muß aus dem gleichen Grunde abgelehnt werden. Damit stellt sich die Frage, inwieweit der Lebensraum in Beziehung zu den Proportionsverhältnissen stehen könnte. Europa besitzt nur wenige ausgedehntere Flachländer, besonders fehlen solche in seinen mittleren, westlichen und südlichen Teilen; das europäische Berg- und Hügelland war aber zu allen Zeiten des Pleistozäns von zahlreichen und großen Pferdeherden bevölkert. Die Pflanzendecke bestand während der warmen und gemäßigten Klimaphasen aus dichterem oder lockerem Wald, der nur während ausgesprochener Kaltzeiten einer subarktischen bis arktischen Tundra wich. Klimatische Zustände, die eine Ausdehnung der warmtrockenen Grassteppe aus östlicheren Gebieten bis nach Westeuropa gestatteten, waren wahrscheinlich selten und nur von kurzer Dauer. Bergig-hügeliges Gelände, das dazu noch unter Waldbedeckung stand, machen für seine Bewohner eine rasche, ausdauernde, gleichmäßige Schnelligkeit entbehrlich und, was noch wichtiger ist, sie ist bei einem solchen Landschaftscharakter auch gar nicht möglich, ohne die Tiere zu gefährden. In der Tundra wieder zwingen die zahlreichen Sumpfstellen und Wasserlöcher, der breiige Boden während der sommerlichen Auftauperiode zu bedächtigen Bewegungen. Auch Nordasien war während des Pleistozäns wahrscheinlich entweder Waldgebiet oder Tundra, abgesehen davon, daß auch dort Gebirge und Hügelländereien keineswegs fehlten.

In einer entsprechenden Umwelt lebten auch die Arten der Amerhippus-Gruppe, die entweder Wald- oder Gebirgsbewohner waren. Nach Hofstetten (1952) besitzt unter den Zebras das Bergzebra (Hippotigris Hartmannae) verglichen mit den Steppenzebras verkürzte Gliedmaßen. Der Schluß, die schwergebauten, großen und kleinen Equiden mit kurzen, plumpen Metapodien wären Berg-, Wald- und Tundrenbewohner gewesen, während die schlankfüßigen in warm- oder kalttrockenen Ebenen und offenen Landschaften beheimatet waren und in der Gegenwart noch sind, scheint daher nicht zu gewagt. Die Proportionierung fassen wir daher als Ergebnis der folgenden in Wechselwirkung stehenden Faktoren auf: Gelände, Vegetation, Bodenbeschaffenheit, Verhalten (Temperament!), Reaktionsbereitschaft und Tempo bei einem Wechsel der Umweltbedingungen.

Obwohl das Ziel der Untersuchung nicht die Lösung taxionomischer und stammesgeschichtlicher Fragen ist und naturgemäß auch nicht sein kann, seien zu dieser Thematik doch noch einige Bemerkungen skizziert. Das vormindelzeitliche Großpferd scheint eine gut umgrenzbare Einheit zu sein; wenigstens spricht nichts dafür, daß es in diesem Zeitabschnitt zwei sehr verschiedene Großformen dieser Gruppe gegeben hätte. Wenn ein ähnlich proportioniertes Großpferd in späteren Wärmephasen wieder in Mitteleuropa erscheint, so dürfen wir in ihm entweder E. mosbachensis oder einen unmittelbaren Abkömmling vermuten; wir wären sonst zu der Annahme gezwungen, ein Großpferd einer ganz anderen Stammeslinie wäre aus uns bis jetzt ganz unbekannten Gegenden im Holstein bzw. Eem nach Europa gekommen. Wahrscheinlicher wird schon die Auffassung sein, E. mosbachensis als Konservativform, ähnlich wie Palaeoloxodon antiquus und Dicerorhinus kirchbergensis hätte im südlichen Europa bis ins Würm hinein gelebt. Die Frage der 
Beziehungen des Großpferdes der sibirischen Eismeerküste (Jana, Tavda, Holstein?, Eem?) zur jungpleistozänen europäischen Großform ist derzeit noch vollkommen offen. Vermutlich aus dem Osten oder Südosten kommend, sind während des Holsteins übermittelgroße Pferde eingewandert, deren Vorgeschichte und Stellung noch zu klären ist (E. steinheimensis?). Die Belege aus diesem Zeitabschnitt sind überhaupt zu spärlich, um Urteile zu gestatten. Eine neue Welle erscheint im Riß, es sind mittelgroße, aber auch schon etwas kleinere Pferde des Typus Körbisdorf (Abkömmlinge mittelgroßer Holsteinformen?, neuzugewanderte Formen anderer Stammeslinien?). Vermutlich sind kleinere Pferde aus nördlicheren Gegenden schon während des Mindels in Mittel- und Westeuropa erschienen (vgl. Sickenberg 1961). Mittelgroße bis untermittelgroße Pferde sind zumindest vom Riß angefangen in Europa und Nordasien weit verbreitet (E.germanicus, E.chosaricus, E. missi usw.), vielleicht mit einer gewissen Arealschrumpfung im Eem. Artliche, vielleicht sogar unterartliche Identität der jungpleistozänen Pferde in der faunistisch eine Einheit bildenden europäisch-nordasiatischen Region darf teilweise angenommen werden. Sie verschwinden als Wildformen erst im Alt(?)holozän (GROмоva 1949, die altholozänen Vertreter dürften nach LundHolm 1949 domestiziert worden sein). Stammesgeschichte und verwandtschaftliche Beziehungen innerhalb dieser Gruppe, die zweifellos ein Formengemenge darstellt, bedürfen einer Klärung, ebenso sind die Umweltbeziehungen der einzelnen Formen noch zu ermitteln. Die Kleinstpferde (Typus Freyburg b) erscheinen, wenigstens in Europa, erst spät, im Laufe des Würm. Auch in Nordasien sind sie geologisch allem Anschein nach nicht sehr alt. Der Ansicht Gromova's und Lundholm's, jene hätten mit dem Przewalski-Pferd nichts zu tun (Gromova 1949, 1959, Lundholm 1949), darf vorbehaltlos zugestimmt werden. Nach den Proportionen waren es tarpanoide Pferde. Unter dem gesamten europäischen und asiatischen Metapodienmaterial befindet sich kein gesicherter pleistozäner Fund, der auf das mongolische Wildpferd eindeutig sich beziehen läßt. Ob und inwieweit die europäischen und nordsibirischen Kleinstpferde mit dem subrezenten Tarpan identifiziert werden dürfen, bedarf gleichfalls gründlicher Untersuchungen, sicher ist nur, daß sie nicht, wie vielfach üblich, die Bezeichnung E. Przewalski PoL. tragen dürfen.

\section{Anhang}

Zusammenstellung der in den vorliegenden Arbeiten genannten Fundorte :

1. Mittel- und Westeuropa

Achenheim (Elsaß): Lösse, Schwemmlösse, verschwemmte Böden auf der Terrasse von Achenheim. Das Profil reicht vom Holstein (Mindel) bis zum Würm II + III. In den meisten Schichten Pferdereste, von WerNerT 6 verschiedenen Arten zugeteilt (E. robustus, E. steinheimensis, E. germanicus, E. przewalski, E.gmelini, E.(Asinus) bydruntinus). Die Reste der großwüchsigen Pferde werden unter der Bezeichnung „E. robustus $^{\text {" }}$ zusammengefaßt; sie sind auf den älteren Abschnitt der Schichtfolge (HolsteinRiß) beschränkt. Temperatur warm bis gemäßigt. Echt kaltzeitliche Faunen treten erst in den höheren Teilen des Profiles auf (Würm). Die Pferdereste sind hier kleiner (WERNERT 1957, Maße eben daher).

Aschenstein b. Freden (Niedersachsen, Leinetal). Blockhalde mit lehmiger Ausfüllung der Zwischenräume (Bergsturzmassen); Jagdstation, Spätmagdalen = Würm III, Temperatur kalt (Eigene Messungen).

Barley (Niedersachsen, Salzgitterscher Höhenzug): Angaben über die Fundschicht und deren Alter liegen nicht vor (SCHWARz 1927).

Burgtonna (Thüringen): Travertin, Eem, warmzeitlich (Maße n. Schwakz 1927).

Datteln (Westfalen): Würm (Woldstedt 1958, Maße n. Schwarz 1927).

Dörstewitz (Thüringen): Keine Angaben (Maße n. Schwarz 1927).

Fontéchevade (Frankreich): Höhlensedimente - Riß? - Eem - Würm. Die Pferdereste stammen aus den riß(?)-eemzeitlichen Ablagerungen (E. caballus n. Arambourg). Nach den Größenunterschieden sind 2-3 Formen vertreten, deren Gleichzeitigkeit nicht sehr wahrscheinlich ist. Temperatur: kühl?, gemäßigt bis warm (Alrmen, Arambourg, SCHREUDER 1957, Maße ebendaher). 
Freyburg a. d. U. (Thüringen): Würmlösse i. d. Lehmgrube d. Ziegelei Gerlach, kaltzeitlich (Lehmann 1923, Maße n. Schwarz 1927).

Görsdorf (Brandenburg): Keine Angaben (Maße n. Schwarz 1927).

Gräfentonna (Thüringen): Travertin, Eem, wärmezeitlich (Maße n. Schwarz 1927).

Halbe (Brandenburg): Keine Angaben (Maße n. Schwarz 1927).

Heiligenstadt (O'́sterreich): Löß und Sumpflöß (= humose Schichten), Alter umstritten, nach KüPPER (1955 und briefl. Mitteilung) Würm I, nach ANTONıUs wahrscheinlich älter (1929); höchstwahrscheinlich aber nicht älter als Eem, Temperaturverhältnisse unklar; im Heiligenstädter-Nußdorfer Lößkomplex auch Anzeiger günstigerer Klimaverhältnisse (Dicerorbinus spec.).

Kalla b. Mainz: „Sandlöß“ (n. Schwarz 1927) = Würm?, Holozän? (Maße n. Schwarz 1927).

Koburg (Bayern): Keine Angaben (Maße n. Schwarz 1927).

Königswusterhausen (Brandenburg): Rixdorfer Horizont d. Ziegelei Neu-Kamerun, s. b. Rixdorf.

Körbisdorf (Thüringen): Kiese der Körbisdorfer Terrasse d. Unstrut-Saalehauptterrasse = Riß; Funde besonders aus Kiesgrube Otto b. Körbisdorf; Temperatur gemäßigt bis kühl (LehmanN 1923) (Maße n. Schwarz 1927).

Körbiskrug (Brandenburg): Rixdorfer „Horizont“, s. Rixdorf (Maße n. Schwarz 1927).

Kreuzberg (Brandenburg): s. o.

Lauchstedt (Thüringen): Keine Angaben (Maße n. Schwarz 1927).

Lengefeld (Thüringen): Kiese d. Saaleterrasse = Riß I (n. Voelker 1935). Die Kiese führen aber vorwiegend Reste einer gemäßigten bis warmen Fauna (s. auch Dietrich 1958) (Maße n. Schwarz 1927).

Lingolsheim (Elsaß): Kiese d. Niederterrasse = Würm; kaltzeitlich (WERNERT 1957). (E. przewalski od. E. gmelini n. Bestimmung Wernert 1957, Maße ebendaher).

Mainz-Kastell (Hessen): „Sandlöß“ (Schwarz 1927) = Würm?, Holozän? (Maße n. Schwarz 1927).

Mittenwalde (Brandenburg): Rixdorfer „Horizont“ der Lokalität „Machnower Weinberg“, s. b. Rixdorf (Maße n. Schwarz 1927).

Mosbach (Hessen): Das Alter der Mosbacher Sande reicht von Günz (?) bis Mindel; die Hauptmasse der Sande ist in die Cromer-Warmzeit zu stellen (Woldstedt 1958), die Pferdereste sind nicht horizontiert aufgesammelt, die Zugehörigkeit zu nur einer Form bleibt daher zweifeihaft. Temperatur gemäßigt bis warm (Maße n. v. REICHENAU 1915 u. Schwarz 1927).

Niederlehme (Brandenburg): Rixdorfer "Horizont" s. Rixdorf.

Ofnet (Württemberg): Höhlenablagerungen. Würm II + III, kaltzeitlich (Maße n. Schwarz 1927).

Oreston Cave (England): Keine Angaben (Maße n. Schwarz).

Pfeddersheim (Rheinl.-Pfalz): Lösse, Würm I, II und III, kaltzeitlich (WEILER 1937, E. germanicus, E. Przewalski, Maße ebendort).

Phoeben (Brandenburg): Limnische Schichten, Eem, warmzeitlich, (vgl. Woldstedt 1958, Maße n. SChWARZ 1927).

Pössneck, Ơpitzer Berg (Thüringen): Angaben fehlen (Maße n. Schwarz 1927).

Quedlinburg (Sachsen-Anhalt): Wahrsch. von der Lok. Seveckenberg stammend; Spaltenfüllungen im Gips; höchstwahrscheinlich Würm (Maße n. Schwarz 1927).

Rixdorf (Berlin): Kiese des sog. Rixdorfer „Horizontes" hauptsächlich im SE von Berlin (Fundorte: Königswusterhausen, Körbiskrug, Kreuzberg, Mittenwalde, Niederlehme, Rixdorf), wahrscheinlich in das Interstadial Würm I/II zu stellen. Die Säugetierreste sind ökologisch uneinheitlich, das Klima schwankt während der Bildungszeit zwischen "gemäßigt" bis "sehr kühl“. Auch Umlagerungen aus einer älteren Vollwarmzeit können stattgefunden haben (Dietrich 1932, Maße n. Dietrich 1932, Schwarz 1927).

Roter Berg b. Saalfeld (Thüringen): Spalten- und Taschenfüllungen unterschiedlichen Alters m. ökologisch verschiedenen Gesellschaften in Dolomitstock (Warm- und Kaltfaunen); Alter: Riß (?) - Würm, kaum älter. Material nicht getrennt aufgesammelt, daher ganz uneinheitlich (Maße n. Schwarz 1927).

Saint-Vallier (Frankreich): Lössige Ablagerungen d. Ältestquartärs (Günz oder ältere Kaltzeit); Temperatur gemäßigt bis kühl (?) Maße n. VIRET 1954).

Schussenquelle (Württemberg): Jagdstation, Spätwürm, kühl bis kalt, Maße n. Lundholm 1949).

Solutré (Frankreich): Gehängeschutt, Hauptknochenlager in den mittleren Teilen (Pferde); Interstadial Würm II/III oder Würm III; kühl bis sehr kühl (Maße n. GromovA 1949 u. VIRET 1954).

Sossenheim $=$ Frankfurt-Sossenheim (Hessen): Keine Angaben (Maße n. Schwarz 1927). 
Steeden (Hessen): Höchstwahrscheinlich ist bei SchwArz 1927 unter der Lok. Steeden die "Wildscheuer" b. Steeden gemeint; Höhlenstation, Ablagerungen aus Würm I Würm III umfassend; kühl bis kalt (Maße n. Schwarz 1927).

Steinheim a. d. Murr (Württemberg): Kiese und Sande; Holstein-Riß I, (m. ökologisch verschiedenartigen Faunen, vgl. ADAM 1954); Temperatur warm - gemäßigt - sehr kühl; Pferdereste hauptsächlich aus den Trogontherii- und Trogontherii-Primigenius-Schottern (Spätholstein? Riß I? gemäßigt bis kühl) Maße n. v. ReIchensu 1915, eigene Messungen) ${ }^{1}$ )

Streckau (Thüringen): Keine Angaben (Maße n. Schwarz 1927).

Thiede (Niedersachsen): Spalten- und Taschenfüllungen in Gipsstock; im wesentlichen Würm II und III; kühl bis kalt; (E.germanicus n. Nehring 1884, Maße n. Schwarz 1927 u. NeHRING 1884).

Türmitz = Kösten b. Türmitz (Böhmen): Keine Angaben (Maße n. Schwarz 1927).

Unkelstein b. Remagen (Rheinland): Löß; jüngerer und älterer Löß vorhanden, der jüngere Löß reichlich knochenführend, nach NeHRING 1884 das Skelett d. Typusexemplares von E. germanicus aus den tiefsten Lößlagen. Alter wahrscheinlich Würm I, Temperatur sehr kühl bis kalt (Maße n. NeHring 1884).

Veltheim, Lok. Steinmühle (Sachsen-Anhalt): Lössige, verlehmte Spaltenfüllung in einem als eemzeitlich angesehenen Süßwasserkalk, der jedoch auch ins Holstein gestellt werden könnte. Spaltenfüllung also entweder Würm I (Verlehmung!) oder Riss. (ScHröDER 1930, Maße n. Schwarz 1927).

Vieselbach (Thüringen): Kiese, Riß nach Soergel (n. Stehlin u. Graziosi 1935); faunistisch wäre auch noch Spätholstein möglich, Temperatur nach Fauna gemäßigt, sicher nicht kalt (E.taubachensis n. v. Reichenau 1915, E. steinheimensis n. Soergel, Maße n. SCHWARZ 1927).

Vogelherd (Württemberg): Höhlenstation, Ablagerungen Würm I - Würm III umfassend, Temperatur kühl bis kalt (LenmanN 1954, E.germanicus, E. przewalski, Maße ebendaher).

Weimar (Thüringen): Travertin, eemzeitlich, warm (Maße n. Schwanz 1927).

Westeregeln (Sachsen-Anhalt): Lössige Ausfüllungen von Spalten und Taschen im Gips; im wesentlichen Würm IJ + III, vielleicht auch noch jüngeres Material (Holozän), kühl bis kalt (E. germanicus n. NeHring 1884, Maße n. Schwarz 1927).

Wunstorf (Niedersachsen): Kiese und Sande: Holstein bis Beginn Riß (?); Fauna ökologisch ungleichartig, warm - gemäßigt - kühl, Pferdereste in den verschiedenen Teilfaunen vorhanden (Veröffentlichung durch den Autor in Vorbereitung).

2. Osteuropa

N. B. In der Arbeit Gromova 1949 sind z. T. Alterseinstufungen der Fundlokalitäten vorgenommen worden, die sich nicht ohne große Schwierigkeiten in das mittel- und westeuropäische Zeitschema einpassen lassen. Versuche einer Angleichung wurden vorgenommen. Sämtliche Maße n. Gromova 1949.

Adji Koba (Krim): Aurignacien (Gromova) = Würm II - III, Temperatur kühl, Steppenfauna mit subarktischem Einschlag.

Borchievo II (Woronescher Distrikt): = Spätmagdalen-Azilien (GroмоvA) = Würm III, kühl gemäßigt, Fauna subarktisch.

Buranovka (Südural): Solutré (Gromova) = Interstadial Würm II/III (?) gemäßigt - kühl, Steppenfauna $\mathrm{m}$. Wald- und subarktischen Elementen.

Griebnievaia (Südural): Ohne Kulturreste, Fauna wie oben, Würm.

Ilskaja (Nordkaukasus): Moustier (Gromova) = Eem? (Woldstedt 1958: Würm I), Temperatur warm ?, Waldfauna.

Kliutschewa (Südural): Solutré (Gromova), s. Buranovka.

Koch-Koba (Krim): Moustier, Temperatur vgl. Adji Koba.

Kodak = Stary Kodak (Dnjepopetrowsker Distrikt): Moustier oder noch älter (GRoмоva) = Würm I oder Spätriß (n. WolDSTEDT 1958 Riß ?), kühl-gemäßigt, Wald- und Tundraformen "gemischt".

Kostenki I (Woronescher Distrikt): Spätaurignac-Solutré (Groмоva) = Würm II (?), kühl kalt, Tundrafauna.

Kotchari II (Südural): Solutré (Gromova) s. Buranovka.

Mysy/Kama (Mittl. Wolgagebiet): Mittelpleistozän (Gromova) = Riß (?), kaum älter: gemäßigt - kühl, Steppenfauna m. leicht subarktischem Einschlag (E.caballus missi).

Novgorod-Severski/Desna (Ukraine): Spätsolutré - Frühmagdalen (Gromova) = Würm III, kühl - kalt, Tundrafauna.

1) Für die Bereitstellung des Materials bin ich Herrn Dr. Adam, Stuttgart, sehr zu Dank verpflichtet. 
Orlovski-Höhle (Südural): Solutré (Gromova) s. Buranovka.

Scholokovo (Dnjepopetrovsker Distrikt): Moustier ? (Gromova) = Eem ? oder Interstadial Würm I/II, gemäßigt, Waldfauna ohne arktischen Einschlag.

Tunguz/Wolga (Mittelrußland): Mittelpleistozän (Gromova) = Riß (?) kaum älter, gemäßigt warm, Fauna ähnlich Mysy, Steppenfauna m. Waldformen (E. caballus chosaricus).

Ust-Katav II (Südural): s. o.

3. Nord-Asien

Anabar, Fluß (Eismeerküste): Jungpleistozän, Fauna subarktisch.

Bugatsch: Keine Angaben.

Jana (Fluß, Eismeerküste): Die Funde sind offenbar sehr verschiedenaltrig, sehr bedeutende Unterschiede in der Größe der Pferde, ebenso im morphologischen Gepräge (GroMova). Die Fauna stammt aus Schichten, die unter dem Bodeneis liegen. Die Fauna umfaßt sowoh! gemäßigtwarme wie arktische Elemente und repräsentiert wahrscheinlich verschiedene klimatische Phasen. Unter den Pflanzen befinden sich Laubbäume (Alnus, Betula alba) und Gräser (Gromova). In Anbetracht der geographischen Lage sind die Fundschichten in ein vollwarmes und in ein kühles Interglazial bzw. Interstadial (geographische Lage!) zu stellen. Da das Bodeneis nach den russischen Geologen sich im Riß gebildet haben soll, wären die Fundschichten älter, also Holstein. Noch ältere Warmzeiten kommen nach dem Artbestand nicht in Frage. Möglich ist aber, daß das Bodeneis in verschiedenen Teilen des nördlichsten Sibiriens und auf den Neusibirischen Inseln (s. u.) unterschiedliches Alter besitzt; dann kämen Einstufungen sowohl ins Holstein wie ins Eem in Frage.

Kotelny (Neusibirische Inseln): Gleiche Verhältnisse wie im Flußgebiet der Jana, nur sind Knochen dort auch in Schichten über dem Bodeneis gefunden worden (Flora mit Moosen, Betula nana, Salix polaris). Als Alter ist daher für diese Schichten ein interglazialer Abschnitt mit sehr kühlen Temperaturen (Eem ?, geographische Lage!) oder ein Interstadial anzusetzen, wenn ihre Bildung nicht überhaupt in das nacheiszeitliche Klimaoptimum fällt.

Kubiekova (Jenisseiufer in der Nähe von K. bei Krasnojarsk, N.-Sibirien): Die Fundschichten sollen einen beträchtlichen Zeitraum, der vom Holstein bis ins Spätwürm reicht, umfassen (Gromova). In den Tabellen unterscheidet Gromova ein: „K. älter“ und ein „K. jünger“, über Begleitfaunen und Temperaturverhältnisse liegen keine Angaben vor.

Taimir-Halbinsel: Spätpleistozän oder Holozän, kalt (E. caballus fossilis), VerestchaGin 1959.

Tavda (Nebenfluß d. Tobols, N.-Sibirien): Jungpleistozän (?).

Tschikoi (Fluß, Transbaikalien): Jungpleistozän.

Unga (Fluß, Irkutzker Region): Jungpleistozän.

Vierkholenskaja Gora (Irkutzker Region): Die Funde stammen teils aus dem Würm, teils aus dem Holozän, wurden aber beim Aufsammeln nicht getrennt gehalten.

\section{Schriftenverzeichnis}

Adam, K. D.: Die mittelpleistozänen Faunen von Steinheim an der Murr (Württemberg). - Quaternaria 1, 1954.

Arambourg, L.: Les gros mammifères de couches tayaciennes - in: La grotte de Fontechévade. Arch. Inst. Pal. Hum., Mem. 29, 1958.

Dietrich, W. O.: Uber den Rixdorfer Horizont im Berliner Diluvium. - Z. D. G. Ges. 84, 1932. - Stetigkeit und Unstetigkeit in der Pferdeentwicklung. - N. Jb. Geol. Pal. B, Abh., 1950.

Dubois, A. \& Stehlin, H. G.: La Grotte de Cotencher, station moustérienne. - Mém. Soc. paléontol. Suisse, 52-53, 1932-33.

Gromova, V.: Histoire des Chevaux (genre Equus) de l'Ancien Monde. - Prem. Part.: Revue et description des formes. - Trav. Inst. Pal. Acad. Sci. USSR. 17, 1949. (Übersetzung i.: Ann. Centre d'Etude et de Documentation Pal., 12, 1955). - - Sur le squelette du Tarpan (Equus caballus gmelini ANT) et d'autres chevaux sauvages actuels. - Bjull. moskovsk. Obshchest. Prirody, Otd biol. 64, 1959.

Herre, W.: Beiträge zur Kenntnis der Wildpferde. - Z. Tierzucht 44, 1939.

HofstetTer, R.: Les Mammifères pleistocènes de la Républiqe de l’Équateur. - Mem. Soc. Geol. France 66, N.S. 31, 1952.

KüPpER, H.: Abschnitt „Wien - Neusiedler See“ in: Beiträge zur Pleistozänforschung in Ơsterreich (Exkursionen zwischen Salzburg und March). - Verh. Geol. Bundesanst. Wien, Sonderh. D, 1955. 
L.enmann, R.: Das Diluvium des unteren Unstruttales. - Jb. d. Halle'schen Verbandes 3, 1922.

L.enmann, U.: Die Fauna des "Vogelherdes“ bei Stetten ob Lontal (Württemberg). - N. Jb. Geol. Pal., Abh. 99, 1954.

Lundholm, B.: Abstammung und Domestikation des Hauspferdes. - Zool. Bidrag, Uppsala, 27, 1947.

Moнr, E.: Das Urwildpferd. - Neue Brehmbücherei, H. 249, A. Ziemser Verlag, Wittenberg, 1959.

Nenring, A.: Fossile Pferde aus deutschen Diluvialablagerungen und ihre Beziehungen zu lebenden Pferden. - Landw. Jb. 13, 1884.

Pirlot, P.: Caballine and zebrine Horses in the Norfolk Forest beds. - Proc. Zool. Soc. London, 118, 1948.

v. Reichenau, W.: Beiträge zur näheren Kenntnis fossiler Pferde aus deutschem Pleistozän. Abh. Hess. Geol. L.-Anst. 7, 1915.

Schröder, H.: Über Rhinozeros mercki und seine nord- und mitteldeutschen Fundstellen. - Abh. preuß. geol. L.-Anst. 124, 1930.

Schwarz, E.: Úber die diluvialen Pferde der Equus caballus-Gruppe. - Jb. preuß. geol. L.-Anst. 48, 1927.

Sickeniserg, O.: Säugetierreste aus den elsterzeitlichen Kiesen von Bornhausen/H. - Geol. Jb, (im Druck).

Stehlin, H. G. \& Grazıosi, P.: Richerche sugli Asinidi fossili d'Europa. - Mém. Soc. Pal. Suisse $56,19.35$.

Thoral, M., Riquet, R. \& Combier, J.: Solutré. - Trav. Lab. Géol. Fac. Sci. Lyon, N. S. $2,1955$.

'Toepfer, V.: Die mitteldiluvialen Säugetiere aus der Saaleterrasse bei Lengefeld. - N. Jb. Min. usw., Beil. Bd. 74, Abt. B., 1935.

Verestchagin: The Mammal remains of the Mammoth age originated from the Taimir Peninsula. Bjul. moskovsk. Obshchest. Prirody, Otd. biol. 64, 1959.

Weiler, W.: Die altsteinzeitlichen Funde von Pfeddersheim. - Notizbl. Hess. Geol. L.-Anst., V.F. H. 18, 1937.

Viret, J.: Le Loess à Bancs durcis de Saint-Vallier (Drôme) et sa Faune de Mammiféres villafranchiens. - Nouv. Arch. Mus. d. Hist. Nat. Lyon 4, 1954.

Wernert, P.: Stratigraphie paléontologiques et prehistoire des sédimentes quaternaires d'Alsace Achenheim. - Mém. Serv. Carte géol. Alsace et Lorraine 14, 1957.

Wolnstedt, P.: Das Eiszeitalter. - 2. Bd. Europa, Vorderasien und Nordafrika im Eiszeitalter 2. Aufl., F. Enke, Stuttgart 1958.

Manuskr. eingeg. 26. 6. 1961.

Anschrift des Verf.: Prof. Dr. O. Sickenberg, Niedersächsisches Landesamt für Bodenforschung, Hannover, Wiesenstraße 1. 